This is the final peer-reviewed accepted manuscript of:

Dieci, R., N. Schmitt, and F. Westerhoff. "Interactions between Stock, Bond and Housing Markets." Journal of Economic Dynamics and Control, vol. 91, 2018, pp. 4370.

The final published version is available online at: http://dx.doi.org/10.1016/j.jedc.2018.05.001

Rights / License:

The terms and conditions for the reuse of this version of the manuscript are specified in the publishing policy. For all terms of use and more information see the publisher's website. 


\title{
Interactions between stock, bond and housing markets
}

\author{
Roberto Dieci, Noemi Schmitt and Frank Westerhoff
}

Working Paper No. 133

January 2018

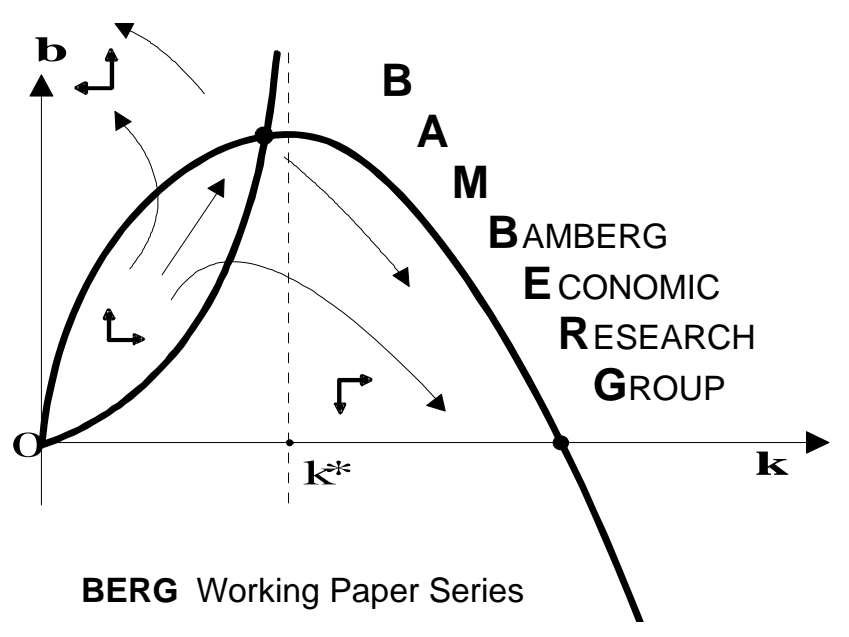

Bamberg Economic Research Group
Bamberg University
Feldkirchenstraße 21
D-96052 Bamberg
Telefax: (0951) 8635547
Telephone: (0951) 8632687
felix.stuebben@uni-bamberg.de
//www.uni-bamberg.de/vwl/forschung/berg/

ISBN 978-3-943153-54-5 


\section{Redaktion:}

Dr. Felix Stübben*

* felix.stuebben@uni-bamberg.de 


\title{
Interactions between stock, bond and housing markets*
}

\author{
Roberto Dieci ${ }^{a}$, Noemi Schmitt ${ }^{b}$ and Frank Westerhoff ${ }^{b}$
}

\begin{abstract}
We develop a model in which investors can participate in stock, bond and housing markets. Investors' market entry decisions are subject to herding effects and depend on the markets' price trends and on their mispricings. The dynamics of our model is governed by a four-dimensional nonlinear map and its unique inner steady state is characterized by standard present-value relations between dividends, rents and the bond rate. Amongst other things, we show that endogenous stock and housing market dynamics emerge, countercyclical to each other, if investors react strongly to the markets' price trends. Such a cross feedback reflects investors' tendency to transfer their enthusiasm from one speculative market to another.
\end{abstract}

JEL Classification: D84; G12; R21

Keywords: Stock markets; housing markets; bond markets; bounded rationality; market interactions; nonlinear dynamics

${ }^{a}$ Department of Mathematics and School of Economics, Management and Statistics, University of Bologna, Italy

${ }^{b}$ Department of Economics, University of Bamberg, Germany

${ }^{*}$ Presented at the 22nd Annual Workshop on Economic Science with Heterogeneous Interacting Agents (WEHIA 2017), Milano, June 12-14, 2017, and at the 23rd International Conference on Computing in Economics and Finance (Invited Session in Honor of Carl Chiarella), New York City, June 28-30, 2017. We thank the participants, in particular Herbert Dawid, Tony He, Cars Hommes, Christian Proaño, Willi Semmler and Jan Tuinstra, for their valuable feedback and comments. The paper also benefited from many helpful suggestions of three anonymous referees. 


\section{Introduction}

Stock and housing markets have historically produced significant boom-and-bust dynamics. Since these turbulences have dramatic effects on the real economy, it is crucial to improve our understanding of what may cause them and, in particular, of the forces and mechanisms governing the interplay of stock and housing markets. In spite of the obvious importance of this issue, the existing empirical literature on the linkages between stock and housing markets is still limited and provides mixed findings. While most early studies typically report low positive (or even negative) correlations, which entails significant opportunities for portfolio diversification (see, e.g. Gyourko and Keim 1992, Hoesli and Hamelink 1997, Fu and Ng 2001), a number of more recent studies indicate that co-movement and causality between stock and housing returns tend to evolve over time and exhibit dependence on the selected time horizon (Okunev et al. 2000, Liow 2006, 2012, Li et al. 2015). Shiller (2015) also reports little relation historically between price changes in the stock and housing market in the US. However, empirical evidence by Borio and Mc Guire (2004) indicates that, internationally, home price booms show an average tendency to begin and to peak a couple of years after stock market booms. Moreover, Shiller (2015) notes that stock market booms display an increasing tendency to alternate with housing market booms, which raises the possibility of a growing (negative) cross feedback between stock and housing markets over long horizons. While it may not be very surprising that a home price boom may begin a few years after a stock market boom - people may feel wealthier and spend more on their homes, thus driving up home prices - Shiller (2015, p. 95) writes that "But it is challenging to envision a feedback model that has home prices rising rapidly even after stock prices are sharply falling. It may seem unlikely that we will ever understand such a phenomenon."

The goal of our paper is to explain why stock and housing markets may display endogenous boom-and-bust dynamics and why the nature of these dynamics may be countercyclical. In doing so, we hope to offer new insights into how these markets function, how they interact and how they may be better regulated in the future. Fortunately, Shiller (2015) provides useful information on how to develop such a feedback model. Most importantly, he discusses questionnaire evidence according to which investor enthusiasm may be transferred from a decreasing stock market to an increasing housing market or from a decreasing housing market to an increasing stock market, thereby intensifying the bust in the decreasing market and amplifying the boom in the increasing market. In Shiller's (2015, p. 96) words: "The drops in the stock market in 2000-2003 had just gotten people increasingly fed up with the stock market and ready to transfer their affections to another market, one that they increasingly believed was the best investment for them", making clear that "There was a sort of cross feedback from the stock market to the housing market, and that must account for a good part of the housing boom that we saw".

But how do investors select markets? As is well known, research in behavioral finance indicates that the typical investor's decision about how much to allocate to stock, bond and housing markets tends not to be based on careful calculations. Instead of holding a well-balanced portfolio, investors seem to concentrate on a rather limited number of assets. Shiller (2015) even argues that investors tend to think in terms of what the 'best investment' opportunity may be for them, and, equally relevant, that they act accordingly. Moreover, the confidence an investor experiences with respect to an investment opportunity depends strongly on his past experience. 
In particular, Shiller (2015) reports that investors regard stock markets as the 'best investment' opportunity if stock markets boom while they lose confidence in stock markets if stock prices decline. This relation establishes a positive feedback process. ${ }^{1}$ But investors are not irrational; they strive to do the right thing. Given their limited cognitive abilities, however, investors tend to follow certain modes of behavior. Shiller (2015) points out that investors use quantitative anchors that show them whether a market is overvalued or undervalued and whether it is time to enter or exit a market. Dividend-price ratios are a typical quantitative anchor for stock markets. Since quantitative anchors eventually reverse booms and busts, they add a negative feedback to the dynamics. According to Shiller (2015), the same forces are at work in housing markets. He reports a striking correlation between housing prices and investors' opinions about what is the 'best investment' opportunity. When housing prices increase (decrease), the fraction of investors who think that the housing market is the 'best investment' opportunity also goes up (down). This once again establishes a feedback process, where the housing market's price-rent ratio serves as a quantitative anchor.

However, the crucial thing to note is that if investors start to switch between stock and housing markets because one market goes up while the other goes down or because one market is overvalued while the other is undervalued, both feedback loops become intertwined. Indeed, we may then observe investors shifting their enthusiasm from one market to another, thereby establishing a (negative) cross feedback between stock and housing markets. This cross feedback may have two effects. First, investors' switching behavior may trigger and/or amplify endogenous boom-and-bust dynamics in stock and housing markets. Second, investors' switching behavior may engender fluctuations in the two markets that are countercyclical to each other. This phenomenon forms the core of our model.

To be more precise, let us summarize the setup of our model. Investors can participate in stock, housing and bond markets. Stock and housing markets are risky markets, allowing investors to profit from price increases and to earn dividends and rents. Bond markets are risk-free and offer investors a constant rate of return. We assume that investors are boundedly rational. To cope with the complexity of their investment tasks, investors try to find out which of the three markets is the 'best investment' opportunity for them. Following Shiller (2015), we furthermore assume that investors are excited about markets that exhibit positive price trends, although they are also aware that fundamental anchors may eventually become relevant and dampen or reverse market trends. Clearly, investors face a discrete choice problem in which they have to decide whether to enter the stock, housing or bond market. The attractiveness of the stock and housing market depends on price movements and on their fundamental economic condition, while the attractiveness of the bond market is given by the risk-free interest rate. Investors' market entry decisions are based on the markets' relative attractiveness. The higher the relative attractiveness of a market, the more likely is that investors will enter it. However, investors' market entry decisions are also subject to herding effects. Finally, stock prices are positively related to stock demand and, ultimately, to stock market participation. The same is

\footnotetext{
${ }^{1}$ Related to this, Kindleberger and Aliber (2005) conclude that more and more investors enter stock markets during stock market booms because they are afraid of missing exceptional profit opportunities. In fact, feelings of regret are a decisive factor in investor behavior. As pointedly expressed by Kindleberger and Aliber (2005, p. 29): "There is nothing as disturbing to one's well-being and judgement as to see a friend get rich. Unless it is to see a nonfriend get rich." They furthermore conclude that such a momentum effect may increase asset prices further, i.e. that there is a positive feedback process.
} 
true for housing markets, while bond prices remain constant.

The emergence of mutually countercyclical boom-and-bust dynamics in the stock and housing market may be explained as follows. If the stock market starts to increase, more and more investors shift their funds from the housing market to the stock market. As long as the stock market continues to increase and the housing market starts to decrease, investors become even more enthusiastic about the stock market and, simultaneously, more pessimistic about the housing market, which tends to prolong the boom in the stock market and to worsen the bust in the housing market. Eventually, however, the upswing in the stock market and the downswing in the housing market lose momentum. First, this may be because almost all investors are located in the stock market. Second, from a fundamental perspective, the housing market appears increasingly more attractive. Once investors start to exit the stock market and to enter the housing market, stock prices start to tumble and housing prices start to recover, which sets in motion a similar boom of the housing market and decline of the stock market, until investors realize that the housing market is overvalued while the stock market is undervalued. As anticipated by Shiller (2015), what keeps the dynamics alive is investors' tendency to transfer their enthusiasm from one speculative market to another. The main goal of our paper is to build a parsimonious mathematical model that captures this idea and provides insight into why and how stock and housing market movement may be countercyclical with respect to each other.

To achieve this goal within an analytically tractable framework, we model the functioning of the two 'risky' markets in an identical, stylized manner. In particular, we do not take into account important specific features of housing, such as its nature as a physical and illiquid asset, the existence of a flexible supply and the impact of housing consumption (rental market). From a formal perspective, the two risky markets in our model may even be regarded as two (types of) equity markets as well. However, there are two reasons why viewing such markets as the stock and the housing market is a very natural interpretation of our setup. First, in our model, investors focus on the 'best investment' opportunity among the available options, and only a fraction of investors liquidate their current investment and enter the other market in a given period; this entails a medium-term commitment to a specific type of investment and is consistent with the 'illiquid' nature of the wealth invested in the housing market. In this respect, note also that, unlike most heterogeneous-agent asset pricing models, our model is not concerned with short-term speculative trading, as we regard the typical length of a time unit as a quarter. Second, and related to the previous point, our interpretation of the two risky markets is consistent with the main dynamic outcome of the model, namely, the emergence of a negative cross feedback between the two markets. In contrast, in a pure financial market setting with two types of (liquid) assets, or investment funds, investors would exploit and promptly reduce the negative correlations by diversifying among funds and by updating their portfolios at short intervals, thus making such countercyclical movements very unlikely. Therefore, our simplifying assumptions on the housing market are mainly aimed at obtaining clear-cut analytical results on the dynamic behavior of the system. Of course, it is possible to develop more general and flexible models that can take into account the peculiar features of the housing market. One such model is outlined and discussed in Appendix 5. However, such models are necessarily of a higher dimension and can only be investigated using numerical simulation. Remarkably, their numerical analysis confirms the robustness of the main qualitative insights of our paper.

The dynamics of our model is driven by a four-dimensional nonlinear dynamical system, having a unique inner steady state characterized by standard present-value relations between 
dividends, rents and the bond rate. Amongst other things, we establish the subsequent results. If investors react strongly to the markets' price trends, the inner steady state becomes unstable via a Neimark-Sacker bifurcation, resulting in oscillatory dynamics. This bifurcation may also emerge if investors react more sensitively to the markets' relative attractiveness. A Flip bifurcation is also possible, triggering a period-two cycle, if investors' reaction to the markets' fundamental conditions is very strong. Investigations based on our deterministic model furthermore reveal that price fluctuations in the stock and housing market have a marked (mutually) countercyclical tendency, although we may also observe more irregular (chaotic) dynamics. Simulations of a stochastic version of our model show that periods in which stock and housing markets move together may alternate with periods in which stock and housing markets move in opposite directions. Interestingly, both scenarios occur for the same parameter setting.

The remainder of our paper is organized as follows. In Section 2, we review related literature, present the setup of our model, and express it as a nonlinear four-dimensional dynamical system. In Section 3, we derive the model's steady state(s), investigate analytically the local stability conditions of the so-called 'fundamental' steady state, and integrate the insights from the local analysis into a broader numerical investigation of the deterministic dynamic model, focusing on the consequences of the local bifurcations and on the impact of the key behavioral and fundamental parameters on market instabilities. In Section 4, we focus on the effect of interest rate changes in a stochastic version of the model. In Section 5, we conclude our paper and highlight a number of avenues for future research. Proofs of our main results and additional details are summarized in an Appendix.

\section{A behavioral model for stock, bond and housing mar- kets}

In this section, we propose a behavioral model to improve our understanding of the complex interplay between stock, bond and housing markets. In Section 2.1, we review related literature. In Section 2.2, we present the setup of our model. In Section 2.3, we finally show that our model can be expressed as a four-dimensional nonlinear dynamical system.

\subsection{Related literature}

Our paper belongs to a blossoming literature stream that explains the dynamics of speculative markets by the behavior of heterogeneous, boundedly rational and interacting agents. For surveys of this line of research, see, for instance, Chiarella et al. (2009), Hommes and Wagener (2009) and Lux (2009). The pioneering contributions by Day and Huang (1990) and Chiarella (1992) already reveal that speculative markets in which agents follow nonlinear technical and fundamental trading rules may be prone to endogenous dynamics. Moreover, the models by Lux (1995) and Brock and Hommes (1998) are examples where agents make discrete choices between technical and fundamental trading rules to determine their investment behavior. Chiarella et al. (2005), Westerhoff and Dieci (2006), Chiarella et al. (2007) and Schmitt and Westerhoff (2014) extend the analysis by considering that agents can also switch between different financial markets. Note that most of these models seek to explain the daily behavior of financial markets. 
In contrast, our goal is to capture a number of important long-term relations between stock, bond and housing markets.

Recently, very similar ideas have been applied to explain the dynamics of housing markets. In Dieci and Westerhoff (2012, 2016), Kouwenberg and Zwinkels (2014, 2015), Bolt et al. (2014), Eichholtz et al. (2015) and Diks and Wang (2016), housing market investors select between extrapolative and regressive expectation rules to determine the development of housing markets. The dynamics of all these models live from the fact that the market impact of destabilizing technical and stabilizing fundamental trading rules, or of destabilizing extrapolative and stabilizing regressive expectation rules, changes over time. Compared to these models, the dynamics of our model is mainly driven by investors' market entry and exit behavior.

There are a few related papers that explore the dynamic consequences of agents' market entry and exit behavior. In Dieci and Westerhoff (2010), firms can supply their products in one of two markets. If firms switch quickly between these two markets, endogenous dynamics may set in. Similar market interaction frameworks are considered in Tuinstra et al. (2014) and Schmitt et al. (2017). What the latter two models have in common with our model is that they apply (exponential) replicator dynamics to determine the number of agents active in a certain market. The model by Schmitt and Westerhoff (2016) is presumably the most closely related model to ours. Considering a setup in which stock market participation depends on current market movements and on the fundamental state of the market, they show that the repeated inflow and outflow of stock market investors increases the amplitude of bubbles and crashes. However, our model is the first model in this line of research that focuses on the dynamic interaction between stock, bond and housing markets.

\subsection{Model setup}

Investors have a long-term trading perspective. In this sense, a trading period in our model may represent a quarter of a year. Moreover, we normalize the total number of investors to one. In each period $t$, fractions $N_{t}^{S}$ and $N_{t}^{H}$ of the total number of investors are active in the stock market and the housing market, respectively, while the residual proportion $N_{t}^{B}=1-N_{t}^{S}-N_{t}^{H}$ is active in the bond market. Rather than assuming that investors select an optimal portfolio from all available investment opportunities in each period, we model agents' investment decisions as a process that takes place in two stages: the decision to enter a specific market, based on a discrete choice principle, and the decision on how much to invest in that market, based on simple behavioral rules. By taking this approach, we adhere to the view that investors really think in terms of what may be the 'best investment' for them and that they act accordingly, as pointed out by Shiller (2015). Moreover, behavioral rules and discrete choice schemes are often assumed in the literature to be reasonable and simplifying models of investment and market entry decisions of boundedly rational agents who must cope with uncertainty in a complex environment, as recently surveyed by Hommes (2013).

In the first stage, agents select one of three investment opportunities: the stock market, the housing market or the bond market. While the bond market allows investors to earn a risk-free return, investors regard stocks and housing as risky investments, as their total return is not only dependent on constant dividends or rents, but also on unpredictable price movements. Since investors are boundedly rational, they make a discrete choice between such alternative investment opportunities, based on three socio-economic principles: investors react to the stock 
and housing markets' price trends and their fundamental economic conditions but they are also influenced by the investment behavior of other investors. The market entry decision is repeated at the beginning of each period.

In the second stage, once an investment opportunity (i.e. a specific market) has been selected, stock, bond and housing market investors decide how much they wish to allocate to stocks, bonds and housing. For simplicity, we assume that agents are ready to invest a fixed amount of money in these markets. Let $I^{S}$ and $I^{H}$ represent the monetary engagements of agents in stock and housing markets, $P_{t}^{S}$ and $P_{t}^{H}$ the unit prices of stocks and housing, and $d_{t}^{S}$ and $d_{t}^{H}$ the individual demands of typical stock and housing market investors in period $t$, respectively. It then follows from $d_{t}^{S} P_{t}^{S}=I^{S}$ and $d_{t}^{H} P_{t}^{H}=I^{H}$ that investors' individual stock and housing demands can be represented by isoelastic functions of stock and housing prices, namely:

$$
d_{t}^{S}=I^{S} / P_{t}^{S}, \quad d_{t}^{H}=I^{H} / P_{t}^{H} .
$$

Of course, the total demand for stock and housing (in physical units) amounts to $D_{t}^{S}:=d_{t}^{S} N_{t}^{S}$ and $D_{t}^{H}:=d_{t}^{H} N_{t}^{H}{ }^{2}$

Prices adjust within each period to equilibrate demand and supply. Denote by $X^{S}$ and $X^{H}$ the total supply of stock and housing (in physical units), assumed fixed over time for simplicity. From the market clearing conditions in the stock and housing market, $D_{t}^{S}=X^{S}, D_{t}^{H}=X^{H}$, it follows that:

$$
P_{t}^{S}=\alpha^{S} N_{t}^{S}, \quad \alpha^{S}:=I^{S} / X^{S}>0
$$

and

$$
P_{t}^{H}=\alpha^{H} N_{t}^{H}, \quad \alpha^{H}:=I^{H} / X^{H}>0 .
$$

Therefore, the price in each market in each period turns out to be proportional to market participation, where the proportionality coefficient can be interpreted as the marginal price impact of one additional investor. ${ }^{3}$ Note that equations similar to (2) and (3), with nonconstant price impact coefficients, can be obtained also in the case of time varying (exogenously or endogenously) supply of assets. Appendix A5 outlines a model extension where the stock of housing (as well as the rent) coevolve with the asset prices.

The bond market is regarded as the risk-free, alternative investment option to stock and housing. The risk-free asset (bond) is assumed to be infinitely elastically supplied at a fixed unit price, with constant return equal to $r>0$. Hence, investors' participation and demand in the bond market is considered residually in our model.

Denote by $D$ and $R$ the constant dividend (per share) and rental income (per housing unit) in the stock and housing market, respectively, and let $\rho_{t}^{S}:=\left(P_{t}^{S}-P_{t-1}^{S}\right) / P_{t-1}^{S}$ and

\footnotetext{
${ }^{2}$ Note that individual demand functions (1) can be derived from a fairly general utility maximization framework. Moreover, our results are not affected by whether residual wealth of stock and housing market investors is used for consumption of a numeraire good or allocated to the bond market. Details are available on request.

${ }^{3}$ In reality, the relation between stock and housing markets is driven by behavioral and rational factors. Whilst emphasizing the impact of behavioral factors, Shiller (2015, p. 94-95) also discusses (perceived) wealth effects in this respect. To highlight Shiller's behavioral view on this matter, we abstain from such factors. Since the proportions of investors active in the stock and housing markets are time varying, a proper modeling of wealth effects would thus imply tracking investors' individual wealth levels. Nevertheless, we considered that investors' monetary engagements depend on the stock and housing markets' past price levels. Such wealth extensions tend to weaken the negative correlation between stock and housing markets, yet do not alter the main qualitative insights provided by our analysis.
} 
$\rho_{t}^{H}:=\left(P_{t}^{H}-P_{t-1}^{H}\right) / P_{t-1}^{H}$ stand for the relative price change in the stock and housing market (referred to simply as stock market and housing market 'return'). In period $t$, investors' market entry decisions depend on the attractiveness of the markets in period $t-1$, represented by $A_{t-1}^{S}$, $A_{t-1}^{H}$ and $A_{t-1}^{B}$. The attractiveness of stock and housing markets is modeled as a mixture of an extrapolative component (based on observed price trends) and a regressive component (based on fundamental conditions, as measured by the dividend-price and rent-price ratios):

$$
A_{t}^{S}=f\left(\rho_{t}^{S}\right)+\gamma \frac{D}{P_{t}^{S}}, \quad A_{t}^{H}=f\left(\rho_{t}^{H}\right)+\gamma \frac{R}{P_{t}^{H}},
$$

where $f$ is a strictly increasing function with $f(0)=0$ and $f^{\prime}(0):=\beta>0 .{ }^{4}$ For instance, a large observed trend in the presence of an already very large price-dividend ratio makes a market less attractive to potential new entrants than a similar trend in the presence of a more reasonable price level. Note that $A_{t}^{S}$ and $A_{t}^{H}$ may be interpreted as expected returns of investors who take into account both price momentum and the likelihood of future price corrections. In particular, for $f$ we select a symmetric increasing $S$-shaped function taking values in the interval $(-\kappa, \kappa),{ }^{5}$ which prevents the dynamics of the system from being too sensitive to large returns. While our local stability results do not depend on the choice of such functions, in our numerical simulations we assume: ${ }^{6}$

$$
f(x)=\mu \arctan \left(\frac{\beta}{\mu} x\right), \quad \mu:=\frac{2}{\pi} \kappa .
$$

Note that, in the presence of a constant marginal price impact per investor, returns correspond to the relative changes of investors' proportions, namely:

$$
\rho_{t}^{S}=\frac{N_{t}^{S}-N_{t-1}^{S}}{N_{t-1}^{S}}, \quad \rho_{t}^{H}=\frac{N_{t}^{H}-N_{t-1}^{H}}{N_{t-1}^{H}} .
$$

Consistent with our assumptions, the attractiveness of the bond market is fixed at the level $A_{t}^{B}=A^{B}=\gamma r$, for any $t$.

Investors' market entry decisions are mainly based on the 'relative attractiveness' of the markets but also contain a herding component. Using the exponential replicator dynamics (e.g. Hofbauer and Sigmund 1988 and Hofbauer and Weibull 1996), we obtain:

$$
\begin{aligned}
N_{t}^{S} & =\frac{N_{t-1}^{S}}{N_{t-1}^{S}+N_{t-1}^{H} \exp \left[\lambda\left(A_{t-1}^{H}-A_{t-1}^{S}\right)\right]+N_{t-1}^{B} \exp \left[\lambda\left(A^{B}-A_{t-1}^{S}\right)\right]}, \\
N_{t}^{H} & =\frac{N_{t-1}^{H}}{N_{t-1}^{S} \exp \left[\lambda\left(A_{t-1}^{S}-A_{t-1}^{H}\right)\right]+N_{t-1}^{H}+N_{t-1}^{B} \exp \left[\lambda\left(A^{B}-A_{t-1}^{H}\right)\right]}, \\
N_{t}^{B} & =\frac{N_{t-1}^{B}}{N_{t-1}^{S} \exp \left[\lambda\left(A_{t-1}^{S}-A^{B}\right)\right]+N_{t-1}^{H} \exp \left[\lambda\left(A_{t-1}^{H}-A^{B}\right)\right]+N_{t-1}^{B}},
\end{aligned}
$$

\footnotetext{
${ }^{4}$ In fact, our steady state and stability results hold under far more general specifications of the attractiveness functions, as shown in Appendices A2 and A3.

${ }^{5}$ Asymmetric smooth versions of such functions can also be obtained, with values in the range $\left(-\kappa_{l}, \kappa_{u}\right)$.

${ }^{6}$ For instance, simulations under the alternative specification $f(x)=\kappa \beta x /\left(\kappa^{2}+\beta^{2} x^{2}\right)^{1 / 2}$ yield qualitatively similar results to those illustrated in this paper.
} 
where $N_{t}^{B}=1-N_{t}^{S}-N_{t}^{H}$, and $\lambda>0$ represents the intensity of choice parameter. Note that (5) to (7) state the following. First, the higher the past relative attractiveness of a market, the more investors will enter it. Second, an increase in investors' intensity of choice implies that more investors enter the market that is relatively more attractive. Third, investors also imitate the behavior of other investors. For instance, a high (low) stock market participation in period $t-1$ tends to lead to a high (low) stock market participation in period $t$, even if the relative attractiveness of the stock market is below (above) the relative attractiveness of the other markets. While Shiller (2015) stresses the relevance of price trends and market fundamentals for investors' market selection process, Hong et al. (2004) argue that a 'social' investor regards

a market as more (less) attractive when more (fewer) of his peers participate in it. ${ }^{7}$ Recent applications of exponential replicator dynamics include Dindo and Tuinstra (2011), Kopel et al. (2014), Tuinstra et al. (2014), Bischi et al. (2015) and Schmitt et al. (2017). ${ }^{8}$

\subsection{Law of motion}

Thanks to (2), (3) and (4), the 'attractiveness differentials' appearing in (5) and (6) can be rewritten in terms of investors' proportions:

$$
\begin{gathered}
A_{t-1}^{H}-A_{t-1}^{S}=f\left(\frac{N_{t-1}^{H}}{N_{t-2}^{H}}-1\right)-f\left(\frac{N_{t-1}^{S}}{N_{t-2}^{S}}-1\right)+\gamma\left(\frac{R}{\alpha^{H} N_{t-1}^{H}}-\frac{D}{\alpha^{S} N_{t-1}^{S}}\right) \\
A^{B}-A_{t-1}^{S}=-f\left(\frac{N_{t-1}^{S}}{N_{t-2}^{S}}-1\right)-\gamma\left(\frac{D}{\alpha^{S} N_{t-1}^{S}}-r\right) \\
A^{B}-A_{t-1}^{H}=-f\left(\frac{N_{t-1}^{H}}{N_{t-2}^{H}}-1\right)-\gamma\left(\frac{R}{\alpha^{H} N_{t-1}^{H}}-r\right) .
\end{gathered}
$$

Therefore, the exponential replicator dynamics (5) and (6) define a law of motion for investors' proportions (and prices) of the two markets, via a system of two nonlinear second-order difference equations. By introducing the auxiliary variables $Z_{t}^{S}$ and $Z_{t}^{H}$ for the lagged values of $N_{t}^{S}$ and $N_{t}^{H}$, respectively, and using $N_{t}^{B}=1-N_{t}^{S}-N_{t}^{H}$, the dynamical system can be recast as a system of four first-order difference equations (two of which are nonlinear), in the dynamic variables $N_{t}^{S}, N_{t}^{H}, Z_{t}^{S}, Z_{t}^{H}$, as follows:

$$
\begin{gathered}
N_{t}^{S}=\frac{N_{t-1}^{S} \exp \left(\lambda A_{t-1}^{S}\right)}{N_{t-1}^{S}\left[\exp \left(\lambda A_{t-1}^{S}\right)-\exp \left(\lambda A^{B}\right)\right]+N_{t-1}^{H}\left[\exp \left(\lambda A_{t-1}^{H}\right)-\exp \left(\lambda A^{B}\right)\right]+\exp \left(\lambda A^{B}\right)}, \\
N_{t}^{H}=\frac{N_{t-1}^{H} \exp \left(\lambda A_{t-1}^{H}\right)}{N_{t-1}^{S}\left[\exp \left(\lambda A_{t-1}^{S}\right)-\exp \left(\lambda A^{B}\right)\right]+N_{t-1}^{H}\left[\exp \left(\lambda A_{t-1}^{H}\right)-\exp \left(\lambda A^{B}\right)\right]+\exp \left(\lambda A^{B}\right)}, \\
Z_{t}^{S}=N_{t-1}^{S}, \\
Z_{t}^{H}=N_{t-1}^{H},
\end{gathered}
$$

\footnotetext{
${ }^{7}$ See also Franke (2014) for an interesting discussion on the ability of discrete choice models to capture herding and to describe aggregate sentiment dynamics.

${ }^{8}$ Formally, shares $N_{t}^{S}, N_{t}^{H}$ and $N_{t}^{B}$ can also be interpreted as portfolio weights of an individual investor who distributes her wealth across stock, housing and bond markets.
} 
where

$$
A_{t-1}^{S}=f\left(\frac{N_{t-1}^{S}}{Z_{t-1}^{S}}-1\right)+\frac{\gamma D}{\alpha^{S} N_{t-1}^{S}}, \quad A_{t-1}^{H}=f\left(\frac{N_{t-1}^{H}}{Z_{t-1}^{H}}-1\right)+\frac{\gamma R}{\alpha^{H} N_{t-1}^{H}}, \quad A^{B}=\gamma r .
$$

While the representation of the model by (5)-(10) is useful for discussing the uniqueness of the fundamental steady state (Appendix A2), its alternative specification (11)-(15) in terms of a four-dimensional nonlinear map is more suitable for deriving conditions for local asymptotic stability (Appendix A3). Section 3 investigates the dynamic properties of the baseline deterministic model (11)-(15). Besides being interesting per se, model (11)-(15) may also be regarded as the nonlinear deterministic skeleton of more realistic models that include exogenous noise and fundamental shocks. One such model is discussed in Section 4.

\section{Dynamic behavior of the deterministic model}

In this section, we carry out a thorough analytical study of the steady-state behavior of the model as well as of the impact of the key parameters on the local asymptotic stability conditions (and the possible local bifurcations) of the 'fundamental' steady state (Section 3.1). Moreover, we present some numerical experiments that offer a broader picture of the dynamic impact of the parameters in conditions of instability and in situations in which the model cannot be satisfactorily represented by the linearized system at the steady state (Section 3.2). Finally, we study in more detail the relation between bond market participation and interest rates (Section 3.3). As we will see, for low interest rates all investors exit the bond market and our four-dimensional model turns into a model with dimension two.

\subsection{Steady state and local stability analysis}

Let us denote steady-state quantities with an overbar. Since quantities $A^{H}$ and $A^{S}$ are defined for strictly positive $N^{H}$ and $N^{S}$, no steady-state solution exists where investors' proportions in the stock or housing market are zero. As a consequence, the possibility $N^{B}=1$ is also excluded at the steady state. However, it is possible that $N^{B}=0$, i.e. $N^{H}+N^{S}=1$, at the steady state. Let us first focus our analysis on inner steady-state solutions where also $\bar{N}^{B}>0$, or equivalently $\bar{N}^{S}+\bar{N}^{H}<1$. In Appendix A2, we prove that there is a unique inner steady state and that all markets need to be equally attractive at the steady state, namely, $A^{B}=\bar{A}^{S}=\bar{A}^{H}$, which implies:

$$
\bar{N}^{S}=\frac{D}{\alpha^{S} r}, \quad \bar{N}^{H}=\frac{R}{\alpha^{H} r}, \quad \bar{P}^{S}=\frac{D}{r}, \quad \bar{P}^{H}=\frac{R}{r} .
$$

Therefore, at the inner steady state, the stock price is given by the discounted value of the dividends while the housing price corresponds to the discounted value of the rents. Since the profitability of the stock, housing and bond market is equal, there are no arbitrage opportunities and, in the absence of price trends, investors have no incentive to change markets. Note that, by using (2) and (3), the steady-state proportions can be rewritten as follows:

$$
\bar{N}^{S}=\frac{D X^{S}}{r I^{S}}, \quad \bar{N}^{H}=\frac{R X^{H}}{r I^{H}},
$$


which reveals that the equilibrium proportions (needed to prevent the violation of the above arbitrage conditions) increase with the existing supply of assets (stock or housing) and decrease with the funds that single investors are willing to invest. We denote the inner steady state (16) as the 'fundamental' steady state (FSS henceforth).

Since parameters $D, R, \alpha^{S}, \alpha^{H}$ and $r$ are strictly positive, steady-state proportions and prices $\bar{N}^{S}, \bar{N}^{H}, \bar{P}^{S}$ and $\bar{P}^{H}$ are strictly positive, too. However, for equation (16) to actually represent the inner steady state, condition $\bar{N}^{S}+\bar{N}^{H}<1$ needs to be satisfied, namely:

$$
r>\frac{\alpha^{S} R+\alpha^{H} D}{\alpha^{S} \alpha^{H}}:=r_{l}
$$

A further non-fundamental steady state may also emerge, as discussed in Appendix A1.

Despite the large dimension of the dynamical system, it is possible to provide a complete local stability and bifurcation analysis of the FSS. The results are summarized in the following

Proposition 1 The FSS is locally asymptotically stable in the region of the parameter space defined as:

$$
\gamma<\frac{2}{r}\left(\frac{1}{\lambda}+\beta\right), \quad \beta<\frac{1}{\lambda} .
$$

Moreover, if a change in one of the parameters determines the violation of the second condition in (18) while the first condition is satisfied, a Neimark-Sacker bifurcation of the FSS occurs. If, on the contrary, the first condition in (18) is violated while the second condition is satisfied, a Flip bifurcation occurs.

Proof. See Appendix A3.

Note that, while a Neimark-Sacker bifurcation will normally be observed if parameters $\beta$ or $\lambda$ become large enough, the occurrence of the Flip bifurcation requires that parameter $\gamma$ becomes large enough. Moreover, only four parameters jointly determine the stability conditions and the nature of the bifurcations of the FSS. Figure 1 provides a qualitative representation of the stability region in the space of parameters $\beta, \gamma$. The arrows show the effect of an increase in parameters $\beta$ and $\gamma$, ceteris paribus. Moreover, the left (right) panel also indicates the impact of parameter $\lambda$ (parameter $r$ ) on the stability region. In particular, the effect of increasing parameter $\beta$ (capturing investors' sensitivity to price trends) is that of a NeimarkSacker bifurcation producing quasiperiodic motion around the steady state. Also, increasing parameter $\gamma$ (investors' sensitivity to price-dividend misalignments) ultimately results in a Flip bifurcation, with the system jumping cyclically between two states. ${ }^{9}$ A comparison of the gray and white areas in the left panel shows that a Neimark-Sacker bifurcation may also be the effect

\footnotetext{
${ }^{9}$ Numerical evidence indicates that both the Neimark-Sacker and the Flip bifurcation of the FSS are of the supercritical type, that is, the loss of stability is simultaneous to the birth of a stable closed invariant curve and a stable orbit of period two, respectively, from the fixed point (see, e.g. Kuznetsov 2004 for an exhaustive discussion of supercritical and subcritical bifurcations). However, the Flip bifurcation may be associated with more complicated bifurcation structures. For instance, setting $D=0.0015, R=0.0035, r=0.01, \alpha^{S}=1$, $\alpha^{H}=1, \beta=0.25, \kappa=0.2, \lambda=1$ and increasing $\gamma$ produces a Flip bifurcation at $\gamma=250$. Using different initial conditions reveals that the inner steady state starts to coexist with a high-amplitude period-two cycle from $\gamma=246.5$ onwards. Preliminary investigations suggest that this high-amplitude period-two cycle is born by a saddle-node bifurcation. Similar bifurcation structures are discussed in Schmitt et al. (2017), yet in a much simpler one-dimensional nonlinear map.
} 
(a) increase in $\lambda$

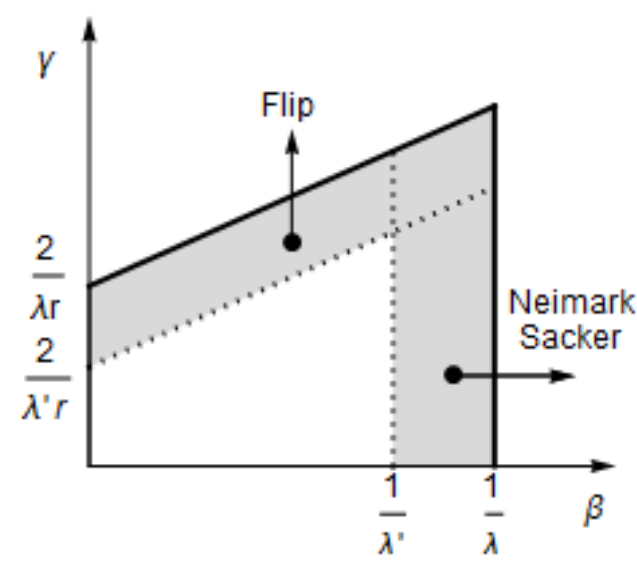

(b) increase in $r$

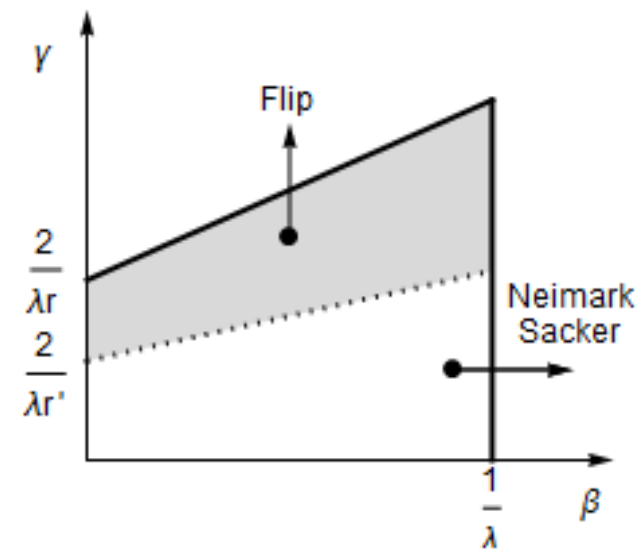

Figure 1: Qualitative sketch of the region of local asymptotic stability of the FSS in the plane of parameters $\beta$ and $\gamma$, for given values of $\lambda$ and $r$. Left panel: stability region for given $\lambda$ and $\lambda^{\prime}\left(\lambda^{\prime}>\lambda\right)$. Right panel: stability region for given $r$ and $r^{\prime}\left(r^{\prime}>r\right)$.

of an increase in the intensity of choice, $\lambda$, for $\beta$ large enough, whereas an increase in $\lambda$ for $\gamma$ large enough may result in a Flip bifurcation. It is interesting to note that, despite the different setup, these results are relatively similar to what we see in a large number of financial market models with chartists and fundamentalists (see, e.g. Westerhoff and Dieci 2006, Chiarella et al. 2009, Dieci and Westerhoff 2013). The gray and white areas in the right panel indicate a somehow less intuitive effect of the interest rate $r$ on the local asymptotic stability of the FSS. First, an increase in the interest rate has no effect on the Neimark-Sacker boundary. Second, an upward movement of $r$ may cause a stability loss through a Flip bifurcation if $\gamma$ is large enough whereas, on the contrary, a downward movement widens the stability region. From an empirical perspective, however, the occurrence of a Flip bifurcation seems to be rather unlikely (see Section 3.2, in particular footnote 10).

\subsection{Nonlinear dynamics and instabilities}

In this section we carry out a numerical investigation of the deterministic model dynamics, complementing the local stability analysis performed in Section 3.1. In particular, we focus on the nonlinear dynamic patterns and cross dependencies that arise for parameter ranges outside the stability domain. The simulations presented in this section are based on a common parameter setting. Unless specified otherwise, the 'fundamental' parameters are given as $D=$ $0.0015, R=0.0035, r=0.01$, which yield the 'fundamental prices' $\bar{P} S=0.15, \bar{P}^{H}=0.35$. Overall, investors have a long-term trading perspective in our paper. In this respect, an interest rate of one percent may be a reasonable assumption for quarterly data. For the behavioral parameters, we select $\alpha^{S}=\alpha^{H}=1$ (from which $\bar{N}^{S}=\bar{P}^{S}, \bar{N}^{H}=\bar{P}^{H}$ ), $\gamma=30, \lambda=1, \kappa=1$ so that the extrapolative component, $f(x)$, in the attractiveness functions becomes (recall that $\left.\mu:=\frac{2}{\pi} \kappa\right): f(x)=\frac{2}{\pi} \arctan \left(\frac{\pi}{2} \beta x\right)$. Parameter $\beta$ is treated as a bifurcation parameter in the 
following analysis. Based on the analytical stability results in Section 3.1, the Neimark-Sacker bifurcation value for parameter $\beta$ is $\beta^{*}=1$. Note that empirical research by Kouwenberg and Zwinkels (2015), Bolt et al. (2014), Hommes (2013) and Hommes et al. (2005) indicates that the trend extrapolation parameter $\beta$ may be larger than 1 . Hence, such a bifurcation scenario is not unrealistic. Although this parameter selection has an illustrative purpose, very similar qualitative results can be observed for wide ranges of the parameters.

As regards the Neimark-Sacker bifurcation for increasing parameter $\beta$ (interpreted as a behavioral sensitivity to recent price trends), Section 3.1 has shown that, while an increase in $\lambda$ makes this bifurcation more likely, an increase in interest rate $r$ has no impact on the NeimarkSacker bifurcation boundary. Figure 2 provides additional information on the impact of such parameters on the dynamic behavior of the system beyond the Neimark-Sacker bifurcation boundary, by means of bifurcation diagrams showing the asymptotic behavior of investors' proportions $N^{S}$ (left panels, in red) and $N^{H}$ (right panels, in blue) for a given initial condition. In all panels, $\beta$ ranges in the interval $(0.5,1.5)$. Under the baseline parameter setting (in particular, $\lambda=1, r=0.01$ ), the top panels show the impact of an increase in $\beta$, visualizing the onset of quasi-periodic dynamics after the Neimark-Sacker bifurcation (taking place for $\beta^{*}=1$ ). The middle panels show the impact of an increase in $\beta$, given a higher value of $\lambda(\lambda=1.5)$ but the same level of $r$ as in the top panels, which confirms that the Neimark-Sacker bifurcation occurs earlier (namely at $\beta^{*}=1 / \lambda=2 / 3$ ) and indicates that the range of fluctuations is wider for any given level of $\beta$. Therefore, the impact of a larger intensity of choice $\lambda$ is both locally and globally destabilizing. Finally, the bottom panels show the impact of an increase in $\beta$, given the same value of $\lambda$ as in the top panels but now a higher interest rate $r(r=0.015)$. While in this case the Neimark-Sacker bifurcation occurs at the same level of $\beta$ as in the top panels, it is remarkable that the asymptotic fluctuations of the destabilized system are tamed. Therefore, while an increase in $r$ has no effect on the local Neimark-Sacker bifurcation boundary, we may say that a higher interest rate is globally stabilizing in the presence of sufficiently large $\beta$ (and d). ${ }^{10}$ Note also that the interest rate increase from $r=0.01$ to $r=0.015$ decreases the steadystate values of stock and housing market investors from $\bar{N}^{S}=0.15$ and $\bar{N}^{H}=0.35$ to $\bar{N}^{S}=0.1$ and $\bar{N}^{H} \approx 0.233$. One reason why higher interest rates may tame the dynamics is as follows. Note first that bond market participation increases with the interest rate, i.e. higher interest rates dry out stock and housing markets. Since investors' market entry behavior is subject to herding effects, participation in these markets tends to remain low once endogenous fluctuations set in.

Figure 3 provides further insight into the model dynamics with an unstable FSS. Each line of panels represents a different scenario, namely the dynamics after the Neimark-Sacker bifurcation (top panels), the dynamics after the Flip bifurcation (middle panels) and an example of chaotic motion (bottom panels). The parameters are as in our baseline selection, with $\beta=1.1, \gamma=30$ in the top panels, $\beta=0.25, \gamma=285$ in the middle panels and $\beta=1.5, \gamma=260$ in the bottom panels. Each plot in the left panels represents both $N^{S}$ (in red) and $N^{H}$ (in blue) in the time domain, while the right panels represent the projection of the attractor in the plane $\left(Z_{t}^{S}, N_{t}^{S}\right)$ and $\left(Z_{t}^{H}, N_{t}^{H}\right)$. Moreover, the dotted lines and crosses indicate the markets' steady-state values.

\footnotetext{
${ }^{10} \mathrm{~A}$ related experiment (not reported here) performed under the Flip bifurcation scenario for fixed $\beta=0.25$ and $\gamma$ ranging in the interval $(235,285)$ shows a destabilizing effect of parameter $\lambda$ similar to that illustrated here. However, a higher interest rate $r$ turns out to be both locally and globally destabilizing.
} 

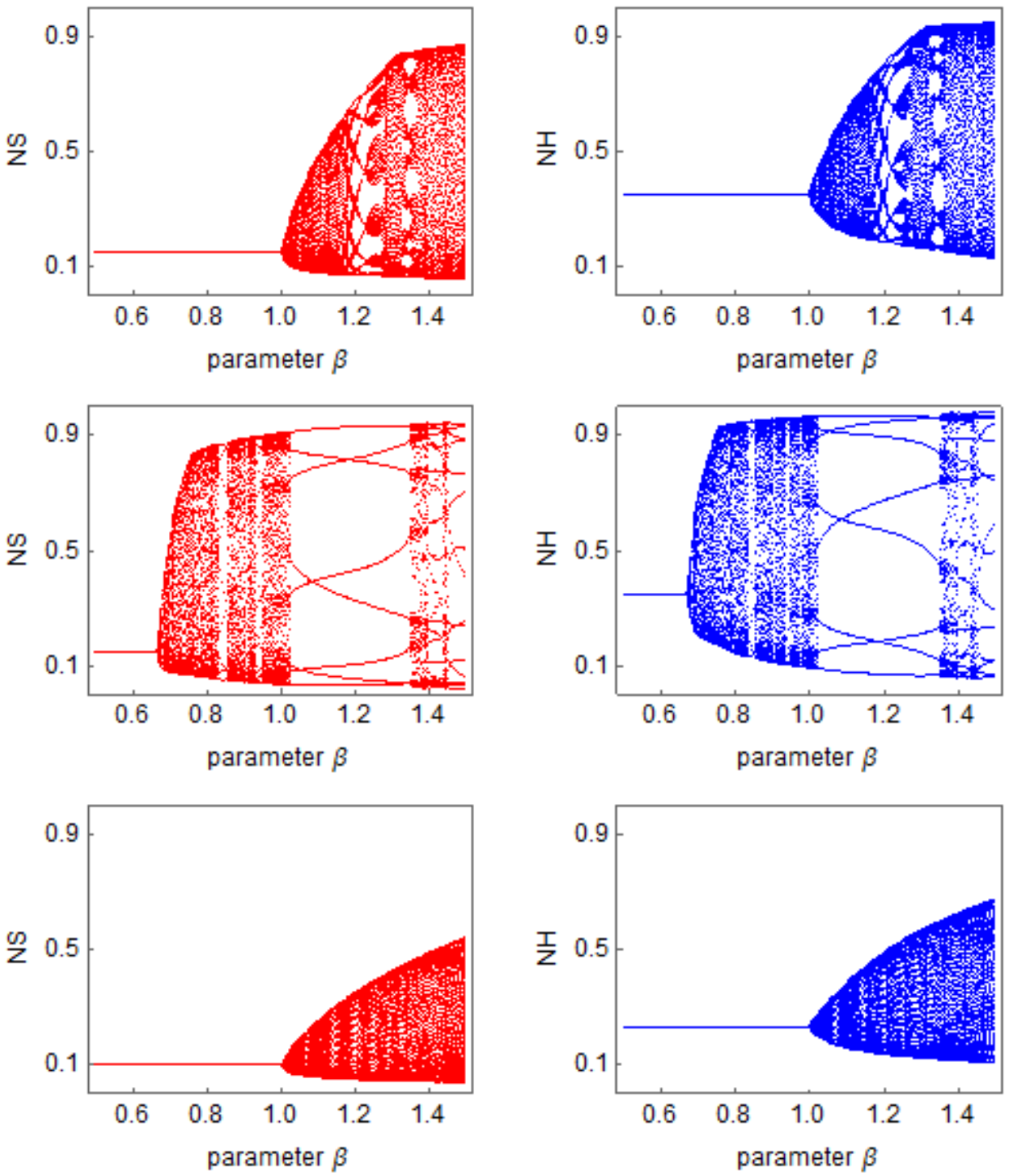

Figure 2: The Neimark-Sacker bifurcation scenario for increasing $\beta$ and the role of parameters $\lambda$ and $r$. The bifurcation plots in the left (right) panels depict the asymptotic behavior of $N^{S}$ or $P^{S}\left(N^{H}\right.$ or $\left.P^{H}\right)$ for $\beta$ ranging from 0.5 to 1.5. Top panels: parameters as in our base selection specified at the beginning of Section 3 (in particular, $\lambda=1, r=0.01$ ). Middle panels: same parameters, except that $\lambda=1.5$. Bottom panels: same parameters as in the top panels, except that $r=0.015$. 
While the dynamic scenario illustrated in the top panels is characterized by quasiperiodic oscillations on a closed orbit, as a consequence of the Neimark-Sacker bifurcation, the middle panels show an example of a cyclical attractor, generated (via a secondary bifurcation) from the attracting two-cycle beyond the Flip bifurcation boundary. In both cases, the time plots reveal stock market movements that are countercyclical to housing market movements. Moreover, in the top panels, the phase plot on the right indicates that large participation (and high prices) in either market in period $t-1$ tends to be followed by large participation in the following period, due to the positive feedback characterizing this parameter regime. In the middle panels, an opposite tendency can be observed, with alternating high and low levels in each market, as implied by the Flip bifurcation. The bottom panels show an example of chaotic motion on a strange attractor. Although the time series in the bottom left panel still indicate countercyclical movements between the two markets, they also indicate - along with the phase plots in the bottom right panel - the existence of more complex dynamics in each market.

Our model reveals that investors' tendency to transfer their enthusiasm from stock markets to housing markets, or vice versa, may set endogenous countercyclical stock and housing market dynamics in motion. Shiller's (2015) prediction is best supported by our model's NeimarkSacker bifurcation scenario. Let us briefly explain the functioning of our model in this case. Consider, for instance, investors switching from the housing market to the stock market. An increase in stock market participation then leads to a higher stock demand and thus elevates stock prices. Simultaneously, the decrease in housing demand depresses housing prices. Other investors perceiving a boom in stock markets and a slump in housing markets may follow their behavior. Stock prices thus increase further while housing prices continue to decline. At some point, however, the markets' momentum ebbs away. First, with the majority of investors already active in the stock market, a further increase in stock market participation is naturally precluded. Second, and presumably more importantly, stock and housing market fundamentals signal to investors that stock markets are overvalued while housing markets are undervalued. Despite investors' imitation behavior, investors will eventually exit stock markets and enter housing markets, causing a reversal in the dynamics. Stock prices then decline while housing prices increase, until stock markets are undervalued while housing markets are overvalued and the markets' developments change direction once again.

In more technical terms, there is a feedback loop operating in each of the two speculative markets, and these feedback loops are intertwined. Both feedback loops have a positive and a negative feedback component. Since increasing (decreasing) stock and housing market participation drives stock and housing markets up (down), investors' market entry and exit behavior constitutes a positive feedback element. However, market fundamentals add a negative feedback to the dynamics. Once markets are sufficiently mispriced, fundamentals cause a reversion of market trends. The (negative) cross feedback between the two feedback loops is due to the fact that investors switch between stock and housing markets. Note that Heemeijer et al. (2009) and Bao et al. (2012) conduct laboratory experiments with single speculative markets and report that speculative markets with positive (negative) feedback structures are more (less) prone to market instability. Given that speculative markets become increasingly connected in reality, it seems worthwhile to extend their experiments, i.e. to test the predictions of our market interaction model in a controlled laboratory environment. 

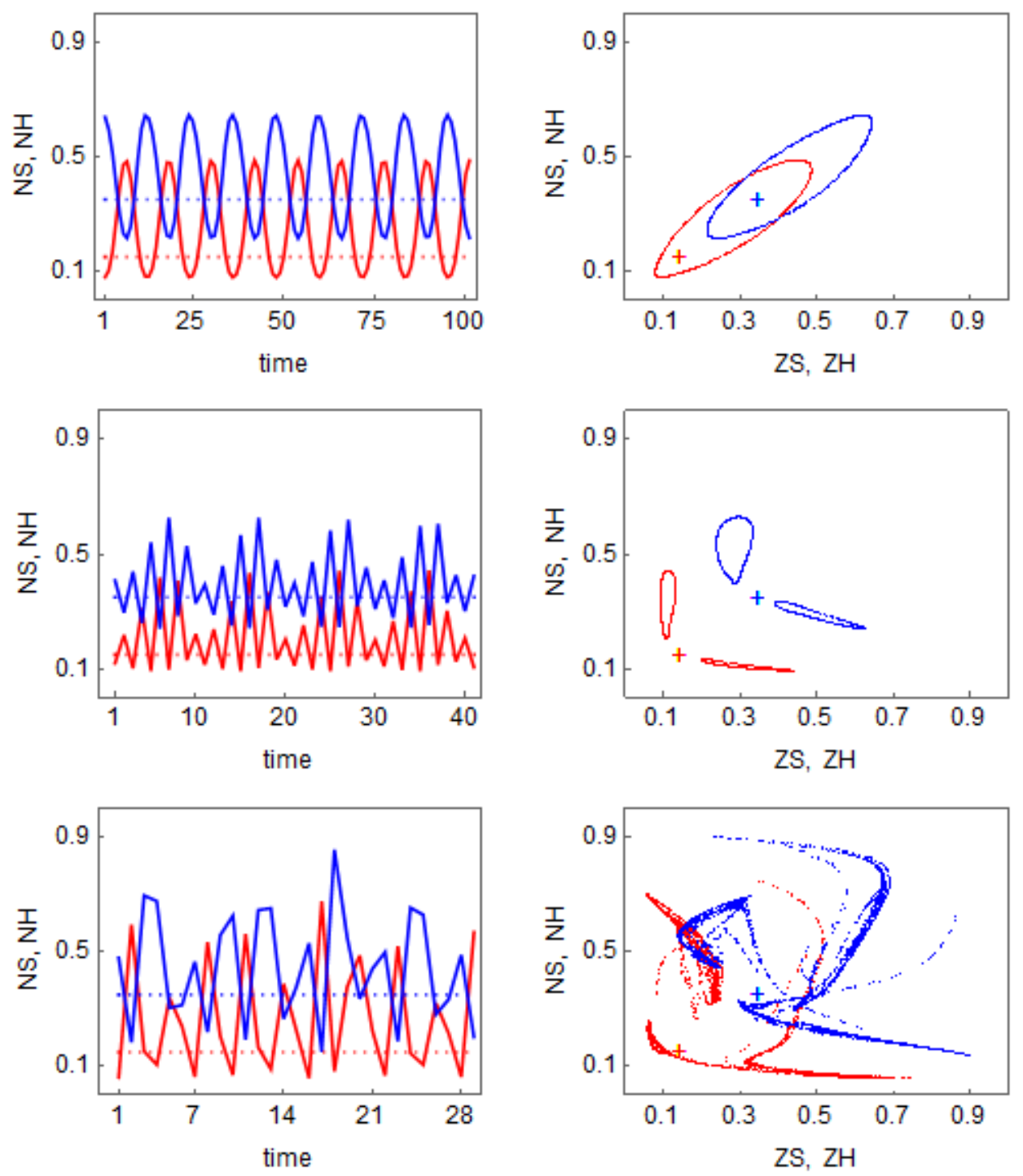

Figure 3: Deterministic dynamics for parameters outside the stability region. The left (right) panels show the dynamics in the time domain (in the phase space) for investors' shares in the stock market (red) and housing market (blue). The dotted lines and crosses indicate the markets' steady-state values. Top panels: dynamics beyond the Neimark-Sacker bifurcation boundary, for $\beta=1.1, \gamma=30$. Middle panels: dynamics following the Flip bifurcation (and a secondary bifurcation), for $\beta=0.25, \gamma=285$. Bottom panels: chaotic dynamics on a strange attractor, $\beta=1.5, \gamma=260$. Other parameters as in our baseline selection. 


\subsection{Interest rates and bond market participation}

Let us briefly discuss the impact of the interest rate (and the role played by the bond market) on the dynamics of the deterministic model. Although the bond market has a fixed return and is treated residually in our model, the constant risk-free rate $r$ plays a substantial role in shaping the dynamics, as visible in Figure 4. In order to illustrate different scenarios associated with the Neimark-Sacker bifurcation over a wide range of interest rate levels (including the case $r<r_{l}$ ), in Figure 4 we use a slightly different parameter setting, namely: $D=0.005, R=0.02$, $\alpha^{S}=\alpha^{H}=1, \lambda=1, \kappa=1, \gamma=20$. For $\beta=0.8$, the bifurcation diagram in the top left panel shows the asymptotic levels of $N^{S}$ (in red), $N^{H}$ (in blue) and $N^{B}=1-N^{S}-N^{H}$ (in black), for $r$ ranging in the interval $(0,0.1)$. Note that the interest rate range also includes very low values, for which the FSS (16) does not correspond to an inner, feasible steady state. Based on Proposition 1 and on Appendix A4, as $\lambda \beta<1$, the steady state is stable for any $r$ in the selected range. As discussed in Appendices A1 and A4, for $0<r<r_{l}=0.025$ the asymptotic dynamics is eventually restricted to the subset $N^{B}=0$ and the steady-state proportions (at the NFSS) are fixed at the levels $\hat{N}^{S}=0.2, \hat{N}^{H}=0.8$, while for $r \geq r_{l}$ investors' shares $\bar{N}^{S}$ and $\bar{N}^{H}$ (at the FSS) tend to decline with the interest rate level. Put differently, if interest rates are high enough, all markets are populated, whereas if interest rates decrease, investors leave the bond market until it is empty. As a consequence, for $0<r<r_{l}$ steady-state prices $\hat{P}^{S}$ and $\hat{P}^{H}$ (here equal to $\hat{N}^{S}$ and $\hat{N}^{H}$, respectively, due to $\alpha^{S}=\alpha^{H}=1$ ) are constant and smaller than their theoretical fundamental values. The bifurcation plot in the top right panel shows a similar situation, for $\beta=1.2$, in which case the dynamics is unstable, with fluctuations around the three steady-state coordinates depicted in the top left panel (except for the empty bond market for low interest rate). This panel clearly illustrates the stabilizing effect of higher interest rates on the fluctuations of the unstable model. In particular, for $r=0.01$ and other parameters as in the top right panel (in particular, $\beta=1.2$ ), the central line of panels illustrates the dynamics of the proportions in the three markets via time plots (left) and phase plots (right). While stock and housing markets display endogenous dynamics, no investors are active in the bond market, that is, the dynamics is restricted to the subset of the phase space such that $N^{S}+N^{H}=1$. In the bottom line of panels, both the time plots (left) and the phase plots (right) depict the dynamics for $r=0.04$ (and other parameters as in the middle panels), with all markets showing fluctuations due to investors' market entry and exit behavior. In this case, the dynamics is no longer restricted to the previous lower-dimensional subset, and a time-varying yet sufficiently large proportion of investors is always active in the bond market.

\section{Stochastic dynamics, interest rates and market co- movements}

Amongst other things, our analysis has revealed the following important insight: starting from a situation in which the model's inner steady state is locally stable, an increase in parameter $\beta$ eventually causes, via a Neimark-Sacker bifurcation, the onset of endogenous countercyclical stock and housing market fluctuations. Although Shiller (2015) argues that stock and housing markets show an increasing tendency to display countercyclical dynamics, the relation between these two markets is less regular in reality than predicted by our model's quasiperiodic behavior. 

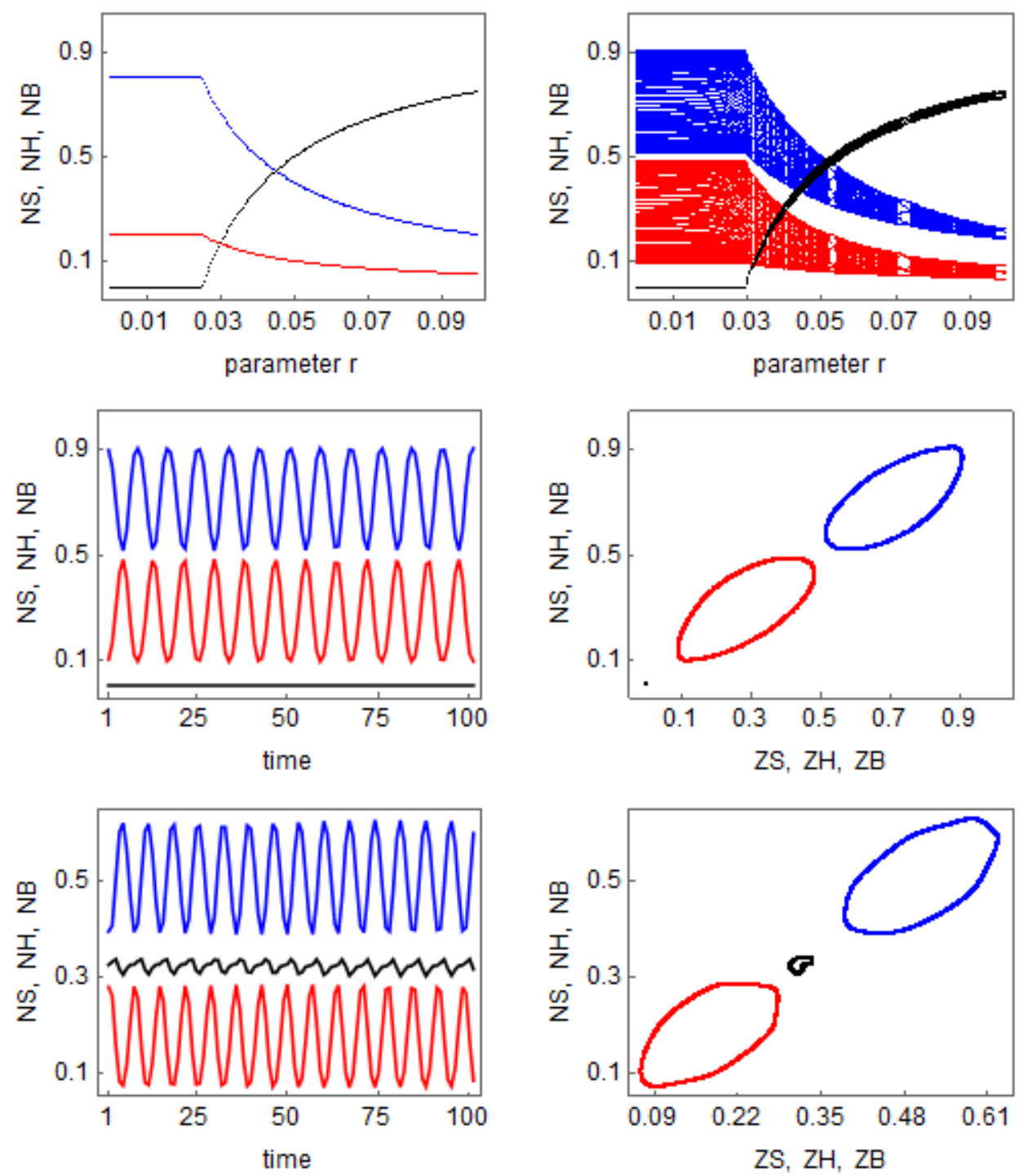

Figure 4: Impact of the interest rate on the asymptotic dynamics in conditions of stability and instability. Parameters as specified at the end of Section 3. Top panels: asymptotic behavior of $N^{S}$ (and $P^{S}$ ), in red, $N^{H}$ (and $P^{H}$ ), in blue, and $N^{B}$ (in black), for $\beta=0.8$ (left) and $\beta=1.2$ (right). Middle panels: dynamics of investors' shares in the three markets via time plots (left) and phase plots (right), for $r=0.01$ and other parameters as in the top right panel (in particular, $\beta=1.2$ ). Bottom panels: dynamics of investors' shares in the three markets via time plots (left) and phase plots (right), for $r=0.04$ (other parameters as in the middle panels). 
The goal of this section is to demonstrate that a stochastic version of our model is able to produce more varied and realistic dynamics. In the presence of exogenous noise (on investors' proportions) and fundamental shocks (to the interest rate), periods in which stock and housing markets move in the same direction may alternate with periods in which they move in opposite directions. Such behavior may arise when the parameter setting is located near the NeimarkSacker bifurcation boundary but still remains inside the inner steady state's stability domain. Stock and housing market prices then tend to track their time-varying fundamental values most of the time, yet exogenous noise may occasionally spark lasting transient countercyclical dynamics. Our analysis reveals that the analytical study of the stability of a model's steady state may prove instrumental to understanding its out-of-equilibrium behavior in the presence of noise. For related work on the intricate interplay between deterministic and random forces, see, for instance, Chiarella et al. (2008, 2011), Dieci et al. (2006), He and Li (2007, 2008) and Hommes (2013).

We modify our model along two lines. First, we consider that exogenous noise may affect the market shares of stock and housing market investors. These random disturbances may reflect errors in investors' decision making processes or further relevant decision factors outside the scope of our model. We integrate exogenous noise in our model by adding two independently and normally distributed random variables with means zero and constant standard deviations $\sigma^{S}=\sigma^{H}=0.005$ to the market shares of stock and housing market investors. The levels of these two exogenous noise components may be deemed as relatively low. Consider a randomly generated one percent change in the market share of stock market investors, say an increase in $N_{t}^{S}$ from 0.25 to 0.26 . Such a change in stock market participation corresponds to a twosigma event and, given the properties of normal distributions, larger shocks can only occur in less than $5 \%$ of cases. Second, we consider that the interest rate follows an AR(1) process, i.e. $r_{t}=r_{t-1}+a^{r}\left(\bar{r}-r_{t-1}\right)+\varepsilon_{t}$, where $\bar{r}=0.01$ denotes the bond market's long-run average interest rate, $a^{r}=0.1$ indicates the interest rate's mean reversion speed, and $\varepsilon_{t}$ is a normally distributed random variable with mean zero and constant standard deviation $\sigma^{r}=0.002$. In a stylized way, such an AR(1) process may be regarded as the result of a central bank's attempt to govern the interest rate in the short run depending on its intended outcomes, whilst targeting a desired long-run interest rate level. ${ }^{11}$

If not stated otherwise, the remaining model parameters are set to $D=0.0025, R=0.0025$, $\alpha^{S}=1, \alpha^{H}=1, \beta=0.97, \gamma=20, \kappa=1$ and $\lambda=1$, respectively. Accordingly, stock and housing markets are structurally symmetric, stock and housing prices are equal to stock and housing market participation and, in a deterministic environment, the model's inner steady state $\bar{N}^{S}=\bar{N}^{H}=\bar{P}^{S}=\bar{P}^{H}=0.25$ would be locally asymptotically stable. Note that we only impose the symmetry assumption to be able to visualize our results more clearly. Apparently, parameter $\beta$ is quite close to the Neimark-Sacker bifurcation boundary. Figure 5 shows a snapshot of the resulting model dynamics, after a longer transient period has been deleted. Assuming that one time step corresponds to one quarter, we encounter a time span of 50 years. The first panel of Figure 5 depicts stock prices (red) and housing prices (blue) in the time

\footnotetext{
${ }^{11}$ Besides reacting to exogenous factors (such as inflation and economic activity), the interest rate process could be (partly) endogenous to the model as long as the central bank takes into account an overheating of financial markets and adopts an interest rate rule that responds to strong misalignments on the markets. This would represent an interesting extension of our setup. We thank an anonymous referee for pointing this out to us.
} 
domain. As can be seen, the dynamics in the first half of the simulation run is markedly different to the dynamics in the second half of the simulation run. In the first 100 periods, stock and housing markets display strong countercyclical fluctuations. Between periods 100 and 200 , stock and housing markets tend to move in the same direction. ${ }^{12}$

The second panel of Figure 5 shows the aggregated number of stock and housing market investors while the third panel shows the path of the interest rate (given in percent). Interest rates are relatively low around periods 25 and 125 . As a result, bond markets appear less attractive and investors tend to enter stock and housing markets. Indeed, there are two pronounced market entry waves. Interestingly, the first market entry wave is associated with countercyclical stock and housing market dynamics while the second market entry wave is not. The fourth panel of Figure 5 shows the ratios between dividends and interest rates, or between rents and interest rates. Since $D=R$, however, both time series overlap exactly. Note that the fourth panel represents the stock and housing markets' short-run fundamental values. By short-run fundamental value we mean in the following the fundamental value that would prevail if interest rates were constant from now on. Whenever interest rates are relatively low (high), stock and housing markets are relatively attractive (unattractive). The market entry waves depicted in the second panel of Figure 5 reveal that investors in fact react to the stock and housing markets' short-run fundamental values, but also that their reaction is rather sluggish. In particular, the sum of stock and housing market investors remains substantially below one, although the stock and housing markets' short-run fundamental values may exceed that level.

To better understand the functioning of our stochastic model, it is helpful to investigate the impact of certain model parts on the dynamics in isolation. Figure 6 is based on the same parameter setting as Figure 5. The top left panel of Figure 6 shows the dynamics when there is neither exogenous noise $\left(\sigma^{S}=\sigma^{H}=0\right)$ nor fundamental shocks $\left(r_{t}=\bar{r}=0.01\right)$. In a deterministic environment, stock and housing markets then generate a countercyclical adjustment towards their steady-state values. Since parameter $\beta$ is close to one, the adjustment process takes quite a while. For the selected initial conditions, we observe significant countercyclical stock and housing market fluctuations even after 100 periods. In the top right panel of Figure 6 , exogenous noise is added to the dynamics, and a larger transient has been deleted. Obviously, exogenous noise keeps the dynamics alive, i.e. it causes permanent countercyclical stock and housing market dynamics. In the center left panel of Figure 6, exogenous noise is substituted by fundamental shocks. Due to changes in the interest rate (depicted in the third panel of Figure 5 ), investors enter stock and housing markets in an almost perfectly synchronized manner and thus stock and housing markets move almost exactly in the same direction. Note that this result does not hinge on the assumption that stock and housing markets are symmetric. In the center right panel of Figure 6, we break the model's symmetry and set $D=0.003$ and $R=0.0025$. As a result, stock market prices are, on average, above housing market prices. Still, both time series are highly synchronized. The bottom left panel of Figure 6 shows the

\footnotetext{
${ }^{12}$ Of course, the duration in which stock and housing markets move in the same or opposite directions is not constant. While the depicted simulation run may be regarded as representative, the feature that both periods roughly comprise 100 periods has been particularly selected for illustrative purposes. Moreover, and this will become clearer in the sequel, decreasing (increasing) $\beta$ creates a stronger (weaker) comoving behavior of stock and housing markets. Note also that with interest rates fluctuating between 0 and 0.02 and $\beta$ being near 1 , the emergence of a flip bifurcation would require values of $\gamma$ above 200. While we can rule out that such a bifurcation scenario occurs here, we recall that higher interest rates tend to tame the model's global dynamics.
} 

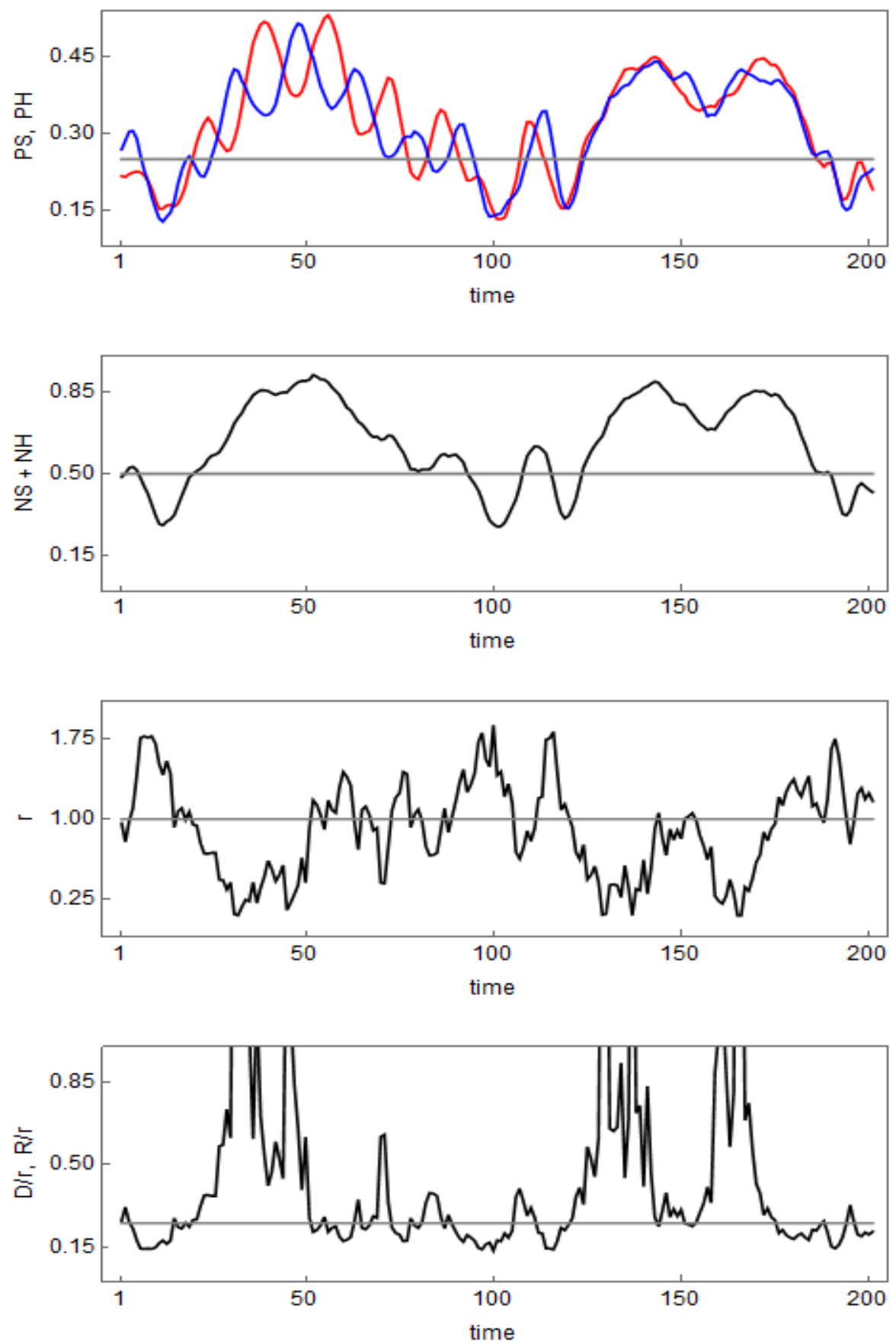

Figure 5: The joint impact of exogenous noise and fundamental shocks. The four panels show from top to bottom (i) the evolution of stock (red) and housing (blue) market prices, (ii) the aggregated market shares of stock and housing market investors, (iii) the interest rate and (iv) the stock and housing markets' short run-fundamental values. The dynamics is depicted for 200 periods; a longer transient has been erased. The underlying parameter setting is reported in Section 4. 
dynamics when exogenous noise and fundamental shocks are jointly present (the dynamics is identical to the one in the top panel of Figure 5). The bottom right panel of Figure 6 reveals what happens if parameter $\beta$ becomes larger than 1. If parameter $\beta$ crosses the Neimark-Sacker bifurcation boundary, the model's inner steady state becomes unstable. Despite comovements in the stock and housing markets' short-run fundamental values, the dynamics is characterized by pronounced countercyclical fluctuations over the entire time range.

To sum up, our model shows that periods in which stock and housing markets move in the same direction may alternate with periods in which they move in opposite directions. These intriguing dynamics are the result of a complex interplay between nonlinear forces, exogenous noise and fundamental shocks. Moreover, the parameterization of our model has to be such that its inner steady state is on the edge of losing its local stability via a Neimark-Sacker bifurcation. The comoving part of the dynamics has a fundamental character. Since the model's inner steady state is locally stable, stock and housing prices track their time-varying short-run fundamental values. For instance, a gradual decrease in the interest rate causes a gradual increase in the stock and housing markets' short-run fundamental values. As investors enter stock and housing markets, both markets are simultaneously driven upwards. Of course, the same argument holds in the opposite direction. Increasing interest rates make the bond market more attractive. Investors exit stock and housing markets, thereby depressing these markets. The countercyclical part of the dynamics has a behavioral character. Exogenous noise may push stock and housing markets further away from their short-run fundamental values and spark a lasting countercyclical adjustment process. If investors start to migrate from housing to stock markets, e.g. because stock prices go up while housing prices go down or because the stock market is undervalued while the housing market is overvalued, stock markets improve while housing markets tumble. Investors' market entry and exit behavior, i.e. their repeated transfer of enthusiasm from one market to another, keeps these countercyclical dynamics alive until fundamental forces regain control over the markets.

\section{Conclusions}

Stock and housing markets have displayed significant boom-and-bust dynamics with severe consequences for the real economy in the recent past. According to Shiller (2015), the dynamics of these markets is driven by investors' market entry and exit behavior. In particular, investors establish a (negative) cross feedback between stock and housing markets by transferring their enthusiasm back and forth between these markets. The goal of our paper is to investigate the functioning of stock and housing markets and the interactions between them. Within our model, investors can participate in stock, bond and housing markets. The bond market guarantees investors a safe return. Investors' market entry decisions depend on the markets' price trends and their mispricings. However, investors are also influenced by the behavior of other investors. Once investors have made a behavioral choice about which of the three markets offers them the 'best investment' opportunity, they determine their demand. The model's market clearing setup implies that stock and housing market prices depend positively on stock and housing market demand and thus on stock and housing market participation. The dynamics of our model is driven by a four-dimensional nonlinear dynamical system. The model's unique inner steady state reveals that stock and housing prices are given by the discounted values of dividends and 

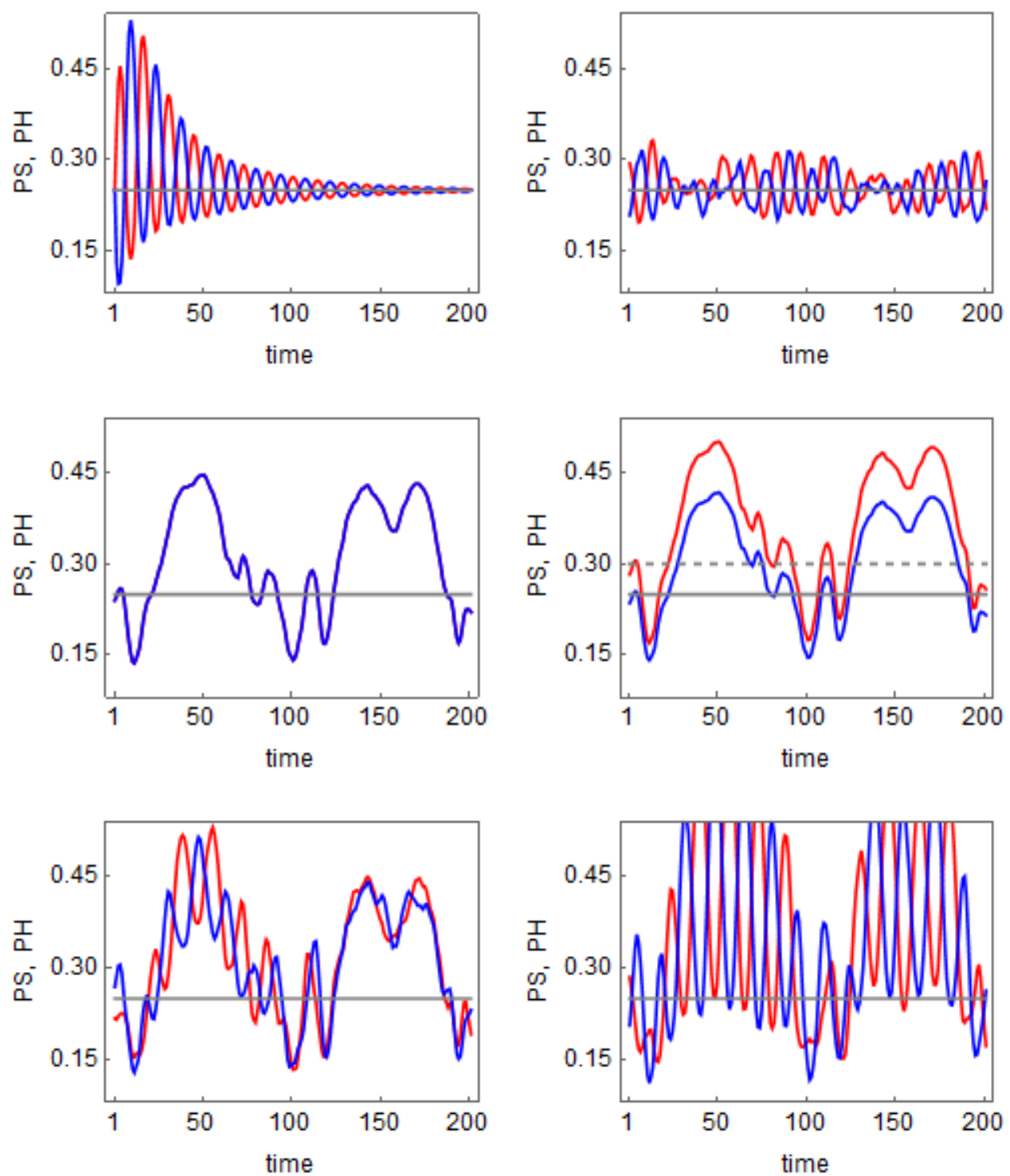

Figure 6: The isolated impact of exogenous noise and fundamental shocks. The six panels show from top left to bottom right the evolution of stock (red) and housing (blue) market prices in (i) the absence of both exogenous noise and fundamental shocks, (ii) in the absence of fundamental shocks, (iii) in the absence of exogenous noise, (vi) in the absence of exogenous noise, assuming that stock and housing markets are asymmetric, (v) with exogenous noise and fundamental shocks, and (vi) with exogenous noise and fundamental shocks, assuming that the inner steady state is unstable. The dynamics is depicted for 200 periods. Except in the top left panel, a longer transient has been erased. The underlying parameter setting is reported in Section 4 . 
rents, respectively. Our stability analysis reveals, amongst other things, that the inner steady state becomes unstable if investors react too strongly to the markets' price trends.

Numerical investigations based on our deterministic model reveal that stock and housing markets are prone to endogenous countercyclical fluctuations. Note that investors who switch from the stock market to the housing market simultaneously dampen the stock market and boost the housing market. Once such a process is set in motion, it may easily persist for a while. Further price declines in the stock market and further price increases in the housing market cause even more investors to turn their attention from the stock market to the housing market. Eventually, investors realize that the stock market is undervalued while the housing market is overvalued. When investors leave the housing market and return to the stock market, stock markets recover and housing markets decline. As predicted by Shiller (2015), the tendency of investors to shift their enthusiasm back and forth between stock and housing markets may cause endogenous countercyclical stock and housing market dynamics. Note that countercyclical dynamics may also emerge if rents and housing supply evolve endogenously, a fact that underlines the robustness of our results.

Simulations of a stochastic version of our model with time-varying interest rates show that periods in which stock and housing markets move in the same direction may alternate with periods in which stock and housing markets move in opposite directions. Parameter settings that may generate such behavior are located close to the Neimark-Sacker bifurcation boundary but still ensure that the inner steady state is locally stable. The basic model dynamics is thus convergent and stock and housing prices approach their fundamental values. However, random shocks may easily push stock and housing prices further away from their fundamental values and cause a prolonged countercyclical adjustment process of stock and housing markets. We have here an example that nicely underlines the relevance of studying analytically and numerically the steady states of nonlinear dynamical systems, their local asymptotic stability and possible deterministic and stochastic out-of-equilibrium processes. Without question, Carl Chiarella was a pioneer in this research area and produced a number of deep insights into the field of nonlinear economic dynamics. In doing so, he not only pushed the frontiers of this research field to new astonishing limits but also provided numerous fruitful and successful research paths for his many pupils and friends.

We conclude our paper by pointing out five avenues for future research. First, we assume that dividends and rents are constant and exogenously given. For the housing market, one could endogenize rents by adding housing consumption and a rental market to the model and consider that the actual stock of housing depends on new construction and housing depreciation. Appendix 5 may be regarded as a first attempt to address this issue. Similarly, one could endogenize dividends by adding a real sector to the model. Second, investors use constant outlay demand functions in the stock and housing market. One could assume that the demand of investors depends more actively on their current wealth level, which would allow including possible wealth effects as a further source of interactions between stock and housing markets. Third, we model investors' behavior in a rather stylized fashion. One could assume that stock and housing markets are also populated by speculators who follow technical and fundamental trading rules to determine their investment positions. Adding such a feature to the model would provide a natural bridge to the literature on heterogeneous interacting agents. While the behavior of investors would then determine the markets' long-term dynamics, the behavior of speculators would be an additional source of short-term fluctuations. Fourth, our baseline 
deterministic model views stock and housing markets as otherwise unrelated markets with no direct linkages. More general versions of our model should account for other direct influences between stock and housing markets due to, for instance, the stock market impact of housingrelated assets and securitizations. Fifth, we have simulated the model dynamics for various parameter settings to illustrate its basic functioning. It would be interesting to bring our model closer to the data. One could try to estimate our model or to test its main predictions in

a controlled laboratory environment. However, the mere fact that investors' market entry and exit behavior constitutes an important amplifying mechanism of the boom-and-bust behavior of stock and housing market dynamics is proof enough that this research area deserves more attention in the future.

\section{References}

Bao, T., Hommes, C., Sonnemans, J. and Tuinstra, J. (2012): Individual expectations, limited rationality and aggregate outcomes. Journal of Economic Dynamics and Control, 36, 1101-1120.

Bischi, G.I., Lamantia, F. and Radi, D. (2015): An evolutionary Cournot model with limited market knowledge. Journal of Economic Behavior and Organization, 116, 219-238.

Bolt, W., Demertzis, M., Diks, C., Hommes, C. and van der Leij, M. (2014): Identifying booms and busts in house prices under heterogeneous expectations. CeNDEF Working Paper 14-13, University of Amsterdam, Amsterdam.

Borio, C. and McGuire, P. (2004): Twin peaks in equity and housing prices? BIS Quarterly Review, 7, 79-93.

Brock, W. and Hommes, C. (1998): Heterogeneous beliefs and routes to chaos in a simple asset pricing model. Journal of Economic Dynamics and Control, 22, 1235-1274.

Chiarella, C. (1992): The dynamics of speculative behavior. Annals of Operations Research, 37, 101-123.

Chiarella, C., Dieci, R. and Gardini, L. (2005): The dynamic interaction of speculation and diversification. Applied Mathematical Finance, 12, 17-52.

Chiarella, C., Dieci, R. and He, X.-Z. (2007): Heterogeneous expectations and speculative behavior in a dynamic multi-asset framework. Journal of Economic Behavior and Organization $62,408-427$.

Chiarella, C., Dieci, R. and He, X.-Z. (2009): Heterogeneity, market mechanisms, and asset price dynamics. In: Hens, T. and Schenk-Hoppé, K.R. (eds.): Handbook of financial markets: dynamics and evolution. North-Holland, Amsterdam, 277-344.

Chiarella, C., He, X.-Z., Wang, D. and Zheng, M. (2008): The stochastic bifurcation behavior of speculative financial markets. Physica A, 387, 3837-3846.

Chiarella, C., He, X.-Z. and Zheng, M. (2011): An analysis of the effect of noise in a heterogeneous agent financial market model. Journal of Economic Dynamics and Control, 35, $148-162$.

Day, R. and Huang, W. (1990): Bulls, bears and market sheep. Journal of Economic Behavior and Organization, 14, 299-329.

Dieci, R., Foroni, I., Gardini, L. and He, X.-Z. (2006): Market mood, adaptive beliefs and asset price dynamics. Chaos, Solitons and Fractals, 29, 520-534. 
Dieci, R. and Westerhoff, F. (2010): Interacting cobweb markets. Journal of Economic Behavior and Organization, 75, 461-481.

Dieci, R. and Westerhoff, F. (2012): A simple model of the speculative housing market. Journal of Evolutionary Economics, 22, 303-329.

Dieci, R. and Westerhoff, F. (2013): On the inherent instability of international financial markets: natural nonlinear interactions between stock and foreign exchange markets. Applied Mathematics and Computation, 221, 306-328.

Dieci, R. and Westerhoff, F. (2016): Heterogeneous expectations, boom-bust housing cycles, and supply conditions: a nonlinear dynamics approach. Journal of Economic Dynamics and Control, 71, 21-44.

Diks, C. and Wang, J. (2016): Can a stochastic cusp catastrophe model explain housing market crashes? Journal of Economic Dynamics and Control, 69, 68-88.

Dindo, P. and Tuinstra, J. (2011): A class of evolutionary models for participation games with negative feedback. Computational Economics, 37, 267-300.

Eichholtz, P., Huisman, R. and Zwinkels, R. (2015): Fundamentals or trends? A long-term perspective on house prices. Applied Economics, 47, 1050-1059.

Franke, R. (2014): Aggregate sentiment dynamics: a canonical modelling approach and its pleasant nonlinearities. Structural Change and Economic Dynamics, 31, 64-72.

Fu, Y. and Ng, L.K. (2001): Market efficiency and return statistics: evidence from real estate and stock markets using a present-value approach. Real Estate Economics, 29, 227-250.

Gyourko, J. and Keim, D. (1992): What does the stock market tell us about real estate returns? Journal of the American Real Estate Finance and Urban Economics Association, 20, 457-485.

He, X.-Z. and Li, Y. (2007): Power-law behaviour, heterogeneity, and trend chasing. Journal of Economic Dynamics and Control, 31, 3396-3426.

He, X.-Z. and Li, Y. (2008): Heterogeneity, convergence and autocorrelations. Quantitative Finance, 8, 58-79.

Heemeijer, P., Hommes, C., Sonnemans, J. and Tuinstra, J. (2009): Price stability and volatility in markets with positive and negative expectations feedback: an experimental investigation. Journal of Economic Dynamics and Control, 33, 1052-1072.

Hoesli, M. and Hamelink, F. (1997): An examination of the role of Geneva and Zurich housing in Swiss institutional portfolios. Journal of Property Valuation and Investment, 15, 354-371.

Hofbauer, J. and Sigmund, K. (1988): The theory of evolution and dynamical systems. Cambridge University Press. Cambridge.

Hofbauer, J. and Weibull, J (1996): Evolutionary selection against dominated strategies. Journal of Economic Theory, 71, 558-573.

Hommes, C. (2013): Behavioral Rationality and Heterogeneous Expectations in Complex Economic Systems. Cambridge University Press. Cambridge.

Hommes, C., Sonnemans, J., Tuinstra, J. and van de Velden, H. (2005): Coordination of expectations in asset pricing experiments. The Review of Financial Studies, 18, 955-980.

Hommes, C. and Wagener, F. (2009): Complex evolutionary systems in behavioral finance. In: Hens, T. and Schenk-Hoppé, K.R. (eds.): Handbook of financial markets: dynamics and evolution. North-Holland, Amsterdam, 217-276. 
Hong, H., Kubik, J. and Stein, J. (2004): Social interaction and stock market participation. Journal of Finance, 59, 137-163.

Kindleberger, C. and Aliber, R. (2005): Manias, Panics, and Crashes: A History of Financial Crises. Wiley, New Jersey.

Kopel, M., Lamantia, F. and Szidarovszky, F. (2014): Evolutionary competition in a mixed market with socially concerned firms. Journal of Economic Dynamics and Control, 48, 394-409.

Kouwenberg, R. and Zwinkels, R. (2014): Forecasting the US housing market. International Journal of Forecasting, 30, 415-425.

Kouwenberg, R. and Zwinkels, R. (2015): Endogenous price bubbles in a multi-agent system of the housing market. PLoS ONE, 10, e0129070.

Kuznetsov, Y. (2004): Elements of Applied Bifurcation Theory, Third Edition. SpringerVerlag. New York.

Li, X.-L., Chang, T., Miller, S.M., Balcilar, M. and Gupta, R. (2015): The co-movement and causality between the U.S. housing and stock markets in the time and frequency domains. International Review of Economics and Finance, 38, 220-233.

Liow, K.H. (2006): Dynamic relationship between stock and property markets. Applied Financial Economics, 16, 371-376.

Liow, K.H. (2012): Co-movements and correlations across Asian securitized real estate and stock markets. Real Estate Economics, 40, 97-129.

Lux, T. (1995): Herd behaviour, bubbles and crashes. Economic Journal, 105, 881-896.

Lux, T. (2009): Stochastic behavioural asset-pricing models and the stylized facts. In: Hens, T. and Schenk-Hoppé, K.R. (eds.): Handbook of financial markets: dynamics and evolution. North-Holland, Amsterdam, 161-216.

Medio, A. and Lines, M. (2001): Nonlinear Dynamics: A Primer. Cambridge University Press. Cambridge.

Okunev, J., Wilson, P. and Zurbruegg, R. (2000): The causal relationship between real estate and stock markets. Journal of Real Estate Finance and Economics, 21, 251-261.

Shiller, R. (2015): Irrational Exuberance, Princeton University Press, Princeton.

Schmitt, N. and Westerhoff, F. (2014): Speculative behavior and the dynamics of interacting stock markets. Journal of Economic Dynamics and Control, 45, 262-288.

Schmitt, N. and Westerhoff, F. (2016): Stock market participation and endogenous boombust dynamics. Economics Letters, 148, 72-75.

Schmitt, N., Tuinstra, J. and Westerhoff, F. (2017): Side effects of nonlinear profit taxes in a behavioral market entry model: abrupt changes, coexisting attractors and hysteresis problems. Journal of Economic Behavior and Organization, 135, 15-38.

Shleifer, A. and Vishny, R.W. (1997): The limits of arbitrage. Journal of Finance, 52, 35-55.

Tuinstra, J., Wegener, M. and Westerhoff, F. (2014): Positive welfare effects of trade barriers in a dynamic partial equilibrium model. Journal of Economic Dynamics and Control, 48, 246264 .

Westerhoff, F. and Dieci, R. (2006): The effectiveness of Keynes-Tobin transaction taxes when heterogeneous agents can trade in different markets: A behavioral finance approach. Journal of Economics Dynamics and Control, 30, 293-322.

Wheaton, W. (1999): Real estate "cycles": some fundamentals. Real Estate Economics, 27, 209-230. 


\section{Appendix}

\section{A1. Existence and role of the 'non-fundamental steady state'}

Based on equation (17), if $r<r_{l}$, the FSS (16) is not feasible. As shown in Appendix A4, the steady-state prices and investors' market shares are then necessarily given by:

$$
\bar{P}^{S}=\frac{D}{r_{l}}=\frac{\alpha^{S} \alpha^{H} D}{\alpha^{S} R+\alpha^{H} D}, \quad \bar{P}^{H}=\frac{R}{r_{l}}=\frac{\alpha^{H} \alpha^{S} R}{\alpha^{S} R+\alpha^{H} D}
$$

and

$$
\bar{N}^{S}=\frac{\alpha^{H} D}{\alpha^{S} R+\alpha^{H} D}, \quad \bar{N}^{H}=\frac{\alpha^{S} R}{\alpha^{S} R+\alpha^{H} D},
$$

respectively, where it is clear that $\bar{N}^{S}+\bar{N}^{H}=1$, i.e. $\bar{N}^{B}=0$. Thus, if $r<r_{l}$, there are no investors in the bond market at the steady state. However, as further discussed in Appendix A4, quantities (19) and (20) may also play a role in the 'normal' case $r>r_{l}$, since they represent the coordinates of a non-fundamental steady state (NFSS), obtained under the restriction of the dynamical system to the two-dimensional subspace of equation $N^{S}+N^{H}=1$ (see equations (37) and (38)). These considerations, along with the analysis of the two-dimensional case in Appendix A4 and the discussion on the impact of $r$ in Section 3.3, allow us to identify three different situations in which the NFSS (20) may come into play. They can be summarized as follows:

(a) For $r>r_{l}$, when the FSS defined by (16) is feasible and interior, equation (20) yields a further, non-fundamental steady state, located on the boundary of the model's state space. The NFSS represents, in particular, the steady state of the restriction of the map (11)-(14) to the 'trapping' subset $\mathcal{S}:=\left(N^{S}, 1-N^{S}, Z^{S}, 1-Z^{S}\right), 0<N^{S}<1,0<Z^{S}<1$ (see Appendix A4 for details).

(b) Equation (20) characterizes the unique steady state of the model in the particular case $r=$ $r_{l}$, when the FSS (16) and the NFSS (20) coincide. Hence the steady-state (fundamental) prices (19) can still be expressed as the standard dividend discount ratios.

(c) If $r<r_{l}$, equation (20) provides the unique feasible steady state of the model. In this case, interest rate $r$ is too low to allow steady-state prices in the stock and housing markets to reflect their fundamental values $D / r$ and $R / r$, respectively, because this would imply a negative proportion of the population of investors in the bond market. As a consequence, asset prices are systematically undervalued in equilibrium in this situation, as illustrated in Section 3.3, Figure 4.

\section{A2. Derivation of the interior 'fundamental' steady state}

We derive the inner steady state of the model and prove its uniqueness under fairly general attractiveness functions, namely:

$$
A_{t}^{S}=f\left(\rho_{t}^{S}\right)+g\left(\frac{D}{P_{t}^{S}}\right), \quad A_{t}^{H}=f\left(\rho_{t}^{H}\right)+g\left(\frac{R}{P_{t}^{H}}\right),
$$


where $f^{\prime}>0, g^{\prime}>0 .{ }^{13}$ This additive specification views the attractiveness $A_{t}$ of an asset or a market as made up of two components. The first term $f$ is positively related to the asset's appreciation in the last period. The second component $g$ is negatively linked to the perceived asset (over)valuation, as measured by the price-dividend ratio. Equation (21) can be interpreted, in particular, as the expected return of investors who care about both momentum and possible mean reversion and is consistent with several heterogeneous-agent asset pricing models, in which the 'market-average' expected return is a combination of trend followers' and fundamentalists' beliefs (see, e.g. Chiarella et al. 2009). Since we consider a further investment opportunity (the bond market) with perfectly elastic supply, constant unit price and constant dividend-price ratio $r$, we specify the constant attractiveness of the bond market in a consistent manner as follows:

$$
A^{B}=f(0)+g(r) .
$$

Note that the line of reasoning and the results shown in this appendix and in Appendix A3 remain true in the case of far more general attractiveness functions, specified as $A_{t}=\Phi\left(\rho_{t}, \zeta_{t}\right)$, with $\Phi_{\rho}>0, \Phi_{\zeta}>0$ (where $\rho$ is an asset's capital gain and $\zeta$ is a generic notation for the dividend-price or rent-price ratio). For consistency, in this case we would set $A^{B}=\Phi(0, r)$.

The dynamical system is represented as:

$$
\begin{aligned}
N_{t}^{S} & =\frac{N_{t-1}^{S}}{N_{t-1}^{S}+N_{t-1}^{H} \exp \left[\lambda\left(A_{t-1}^{H}-A_{t-1}^{S}\right)\right]+N_{t-1}^{B} \exp \left[\lambda\left(A^{B}-A_{t-1}^{S}\right)\right]}, \\
N_{t}^{H} & =\frac{N_{t-1}^{H}}{N_{t-1}^{S} \exp \left[\lambda\left(A_{t-1}^{S}-A_{t-1}^{H}\right)\right]+N_{t-1}^{H}+N_{t-1}^{B} \exp \left[\lambda\left(A^{B}-A_{t-1}^{H}\right)\right]},
\end{aligned}
$$

where the differentials in attractiveness among markets, rewritten in terms of investors' proportions, are as follows:

$$
\begin{gathered}
A_{t-1}^{H}-A_{t-1}^{S}=f\left(\frac{N_{t-1}^{H}}{N_{t-2}^{H}}-1\right)-f\left(\frac{N_{t-1}^{S}}{N_{t-2}^{S}}-1\right)+g\left(\frac{R}{\alpha^{H} N_{t-1}^{H}}\right)-g\left(\frac{D}{\alpha^{S} N_{t-1}^{S}}\right), \\
A^{B}-A_{t-1}^{S}=f(0)-f\left(\frac{N_{t-1}^{S}}{N_{t-2}^{S}}-1\right)-g\left(\frac{D}{\alpha^{S} N_{t-1}^{S}}\right)+g(r), \\
A^{B}-A_{t-1}^{H}=f(0)-f\left(\frac{N_{t-1}^{H}}{N_{t-2}^{H}}-1\right)-g\left(\frac{R}{\alpha^{H} N_{t-1}^{H}}\right)+g(r) .
\end{gathered}
$$

We use an overbar to denote the steady-state levels of the dynamic variables. First, note that quantities $A^{H}$ and $A^{S}$ are only defined for strictly positive $N^{H}$ and $N^{S}$, respectively, which rules out the possibility of a steady-state solution in which the proportion of investors in the stock market or in the housing market is zero. Therefore, $\bar{N}^{S}>0, \bar{N}^{H}>0$ and, from $\bar{N}^{S}+\bar{N}^{H}+\bar{N}^{B}=1$, it follows that $\bar{N}^{S}<1, \bar{N}^{H}<1, \bar{N}^{B}<1$. We cannot exclude the possibility that $\bar{N}^{B}=0$, i.e. $\bar{N}^{S}+\bar{N}^{H}=1$. In the following, we restrict our analysis to interior steady states, such that $0<\bar{N}^{S}, \bar{N}^{H}, \bar{N}^{B}<1$. $^{14}$ By imposing the steady-state condition,

\footnotetext{
${ }^{13}$ In particular, in our paper $g$ is linear and $f(0)=0$.

${ }^{14}$ See Appendix A4 for a discussion of the case $N^{B}=0$.
} 
$\bar{N}^{S}=N_{t}^{S}=N_{t-1}^{S}=N_{t-2}^{S}, \bar{N}^{H}=N_{t}^{H}=N_{t-1}^{H}=N_{t-2}^{H}$, the differentials in attractiveness specified above become:

$$
\bar{A}^{H}-\bar{A}^{S}=g\left(\frac{R}{\alpha^{H} \bar{N}^{H}}\right)-g\left(\frac{D}{\alpha^{S} \bar{N}^{S}}\right)
$$

and

$$
A^{B}-\bar{A}^{S}=g(r)-g\left(\frac{D}{\alpha^{S} \bar{N}^{S}}\right), \quad A^{B}-\bar{A}^{H}=g(r)-g\left(\frac{R}{\alpha^{H} \bar{N}^{H}}\right),
$$

whereas, for $\bar{N}^{S}>0, \bar{N}^{H}>0$, the replicator equations (22)-(23) reduce to:

$$
\begin{aligned}
& \bar{N}^{H} \exp \left[\lambda\left(\bar{A}^{H}-\bar{A}^{S}\right)\right]+\bar{N}^{B} \exp \left[\lambda\left(A^{B}-\bar{A}^{S}\right)\right]=1-\bar{N}^{S}, \\
& \bar{N}^{S} \exp \left[\lambda\left(\bar{A}^{S}-\bar{A}^{H}\right)\right]+\bar{N}^{B} \exp \left[\lambda\left(A^{B}-\bar{A}^{H}\right)\right]=1-\bar{N}^{H} .
\end{aligned}
$$

Below we prove that the three investment options need to be equally attractive at the steady state, namely, $A^{B}=\bar{A}^{S}=\bar{A}^{H}$, which results in a unique interior stationary solution. In fact, no interior steady state is compatible with condition $\bar{A}^{S} \neq \bar{A}^{H}$.

Note first that from $1-\bar{N}^{S}=\bar{N}^{H}+\bar{N}^{B}$ and $1-\bar{N}^{H}=\bar{N}^{S}+\bar{N}^{B}$ one can rearrange (26)-(27), respectively, as follows:

$$
\begin{aligned}
& \bar{N}^{B}\left\{\exp \left[\lambda\left(A^{B}-\bar{A}^{S}\right)\right]-1\right\}=\bar{N}^{H}\left\{1-\exp \left[\lambda\left(\bar{A}^{H}-\bar{A}^{S}\right)\right]\right\}, \\
& \bar{N}^{B}\left\{\exp \left[\lambda\left(A^{B}-\bar{A}^{H}\right)\right]-1\right\}=\bar{N}^{S}\left\{1-\exp \left[\lambda\left(\bar{A}^{S}-\bar{A}^{H}\right)\right]\right\} .
\end{aligned}
$$

Consider equations (28)-(29) and assume that $\bar{A}^{H}>\bar{A}^{S}$. In this case, the right-hand side of (28) is negative, while the right-hand side of (29) is positive. Thus, from the left-hand sides of the same equations, it must be true that $\exp \left[\lambda\left(A^{B}-\bar{A}^{S}\right)\right]<1$ and $\exp \left[\lambda\left(A^{B}-\bar{A}^{H}\right)\right]>1$, that is $\bar{A}^{H}<A^{B}<\bar{A}^{S}$, which contradicts the initial assumption. Symmetrically, assuming $\bar{A}^{H}<\bar{A}^{S}$, one obtains the contradictory conclusion $\bar{A}^{H}>A^{B}>\bar{A}^{S}$ by a similar argument. As a consequence, $\bar{A}^{S}=\bar{A}^{H}$ at the steady state. Moreover, by replacing $\bar{A}^{S}=\bar{A}^{H}$ in (28)-(29), it follows immediately that their common value is equal to $A^{B}$. Finally, setting $\bar{A}^{S}=\bar{A}^{H}=A^{B}$ into (24)-(25) results in the unique inner solution, the so-called fundamental steady state (FSS):

$$
\bar{N}^{S}=\frac{D}{\alpha^{S} r}, \quad \bar{N}^{H}=\frac{R}{\alpha^{H} r},
$$

and therefore, in terms of stock and housing prices:

$$
\bar{P}^{S}=\frac{D}{r}, \quad \bar{P}^{H}=\frac{R}{r} .
$$

\section{A3. Stability analysis of the fundamental steady state (FSS)}

Let us denote variables $N^{S}$ and $N^{H}$ with $x_{1}$ and $x_{2}$ and use $z_{1}$ and $z_{2}$ for their one-period lagged values, respectively. We also use $\alpha_{i}$ and $d_{i}, i=1,2$, for parameters $\alpha^{S}, \alpha^{H}, D$ and $R$, respectively. The structure of the four-dimensional map driving the dynamics is as follows: ${ }^{15}$

$$
\begin{gathered}
x_{1}^{\prime}=F_{1}\left(x_{1}, x_{2}, z_{1}, z_{2}\right), \\
x_{2}^{\prime}=F_{2}\left(x_{1}, x_{2}, z_{1}, z_{2}\right), \\
z_{1}^{\prime}=x_{1}, \\
z_{2}^{\prime}=x_{2} .
\end{gathered}
$$

\footnotetext{
${ }^{15}$ The symbol' here denotes the unit-time advancement operator.
} 
Functions $F_{i}, i=1,2$, characterizing the exponential replicator dynamics, can be represented as

$$
F_{i}\left(x_{1}, x_{2}, z_{1}, z_{2}\right)=\frac{x_{i} w_{i}\left(x_{i}, z_{i}\right)}{x_{1}\left[w_{1}\left(x_{1}, z_{1}\right)-\bar{w}\right]+x_{2}\left[w_{2}\left(x_{2}, z_{2}\right)-\bar{w}\right]+\bar{w}},
$$

where:

$$
\begin{aligned}
w_{i}\left(x_{i}, z_{i}\right) & :=\exp \left[\lambda u_{i}\left(x_{i}, z_{i}\right)\right], \quad u_{i}\left(x_{i}, z_{i}\right):=f\left(\frac{x_{i}}{z_{i}}-1\right)+g\left(\frac{d_{i}}{\alpha_{i} x_{i}}\right), \\
\bar{w} & :=\exp (\lambda \bar{u}), \quad \bar{u}:=f(0)+g(r) .
\end{aligned}
$$

Note first that at the FSS $\bar{x}_{i}=\bar{z}_{i}=d_{i} /\left(r \alpha_{i}\right), i=1,2$, and therefore, in particular:

$$
\frac{d_{i}}{\alpha_{i} \bar{x}_{i}}=r, \quad u_{i}\left(\bar{x}_{i}, \bar{z}_{i}\right)=f(0)+g(r)=\bar{u}, \quad w_{i}\left(\bar{x}_{i}, \bar{z}_{i}\right)=\bar{w}
$$

Moreover, if $V=V\left(x_{1}, x_{2}, z_{1}, z_{2}\right)$ denotes the denominator of (30), one also obtains at the FSS:

$$
V\left(\bar{x}_{1}, \bar{x}_{2}, \bar{z}_{1}, \bar{z}_{2}\right)=\bar{w}
$$

In order to build the Jacobian matrix, it is useful to regard functions $F_{i}$ in equation (30) as composite functions of $x_{1}, x_{2}, z_{1}, z_{2}$, through quantities $w_{i}:=w_{i}\left(x_{i}, z_{i}\right), i=1,2$, namely, $F_{i}\left(x_{1}, x_{2}, z_{1}, z_{2}\right)=\tilde{F}_{i}\left(x_{1}, x_{2}, w_{1}, w_{2}\right)$. Therefore, using the chain rule, one can compute the (total) derivatives of $F_{i}, i=1,2$, with respect to each variable as follows:

$$
\begin{aligned}
& \frac{\partial F_{1}}{\partial x_{1}}=\frac{\partial \tilde{F}_{1}}{\partial x_{1}}+\frac{\partial \tilde{F}_{1}}{\partial w_{1}} \frac{\partial w_{1}}{\partial x_{1}}, \quad \frac{\partial F_{1}}{\partial x_{2}}=\frac{\partial \tilde{F}_{1}}{\partial x_{2}}+\frac{\partial \tilde{F}_{1}}{\partial w_{2}} \frac{\partial w_{2}}{\partial x_{2}} \\
& \frac{\partial F_{1}}{\partial z_{1}}=\frac{\partial \tilde{F}_{1}}{\partial w_{1}} \frac{\partial w_{1}}{\partial z_{1}}, \quad \frac{\partial F_{1}}{\partial z_{2}}=\frac{\partial \tilde{F}_{1}}{\partial w_{2}} \frac{\partial w_{2}}{\partial z_{2}}
\end{aligned}
$$

and, symmetrically:

$$
\begin{aligned}
& \frac{\partial F_{2}}{\partial x_{1}}=\frac{\partial \tilde{F}_{2}}{\partial x_{1}}+\frac{\partial \tilde{F}_{2}}{\partial w_{1}} \frac{\partial w_{1}}{\partial x_{1}}, \quad \frac{\partial F_{2}}{\partial x_{2}}=\frac{\partial \tilde{F}_{2}}{\partial x_{2}}+\frac{\partial \tilde{F}_{2}}{\partial w_{2}} \frac{\partial w_{2}}{\partial x_{2}} \\
& \frac{\partial F_{2}}{\partial z_{1}}=\frac{\partial \tilde{F}_{2}}{\partial w_{1}} \frac{\partial w_{1}}{\partial z_{1}}, \quad \frac{\partial F_{2}}{\partial z_{2}}=\frac{\partial \tilde{F}_{2}}{\partial w_{2}} \frac{\partial w_{2}}{\partial z_{2}}
\end{aligned}
$$

The partial derivatives in the above formulas are given by: ${ }^{16}$

$$
\begin{aligned}
& \frac{\partial \tilde{F}_{1}}{\partial x_{1}}=\frac{x_{2} w_{1}\left(w_{2}-\bar{w}\right)+w_{1} \bar{w}}{V^{2}}, \quad \frac{\partial \tilde{F}_{1}}{\partial w_{1}}=\frac{x_{1}\left(1-x_{1}\right) \bar{w}+x_{1} x_{2}\left(w_{2}-\bar{w}\right)}{V^{2}} \\
& \frac{\partial \tilde{F}_{1}}{\partial x_{2}}=-\frac{x_{1} w_{1}\left(w_{2}-\bar{w}\right)}{V^{2}}, \quad \frac{\partial \tilde{F}_{1}}{\partial w_{2}}=-\frac{x_{1} x_{2} w_{1}}{V^{2}}
\end{aligned}
$$

\footnotetext{
${ }^{16}$ With some abuse of notation, we continue to use $V$ to denote the denominator of functions $F_{i}$ defined in (30), even when $V$ is regarded as a function of $x_{1}, x_{2}, w_{1}, w_{2}$.
} 
and symmetrically

$$
\begin{aligned}
& \frac{\partial \tilde{F}_{2}}{\partial x_{1}}=-\frac{x_{2} w_{2}\left(w_{1}-\bar{w}\right)}{V^{2}}, \quad \frac{\partial \tilde{F}_{2}}{\partial w_{1}}=-\frac{x_{1} x_{2} w_{2}}{V^{2}} \\
& \frac{\partial \tilde{F}_{2}}{\partial x_{2}}=\frac{x_{1} w_{2}\left(w_{1}-\bar{w}\right)+w_{2} \bar{w}}{V^{2}}, \quad \frac{\partial \tilde{F}_{2}}{\partial w_{2}}=\frac{x_{2}\left(1-x_{2}\right) \bar{w}+x_{1} x_{2}\left(w_{1}-\bar{w}\right)}{V^{2}}
\end{aligned}
$$

while in general, for $i=1,2$ :

$$
\begin{aligned}
\frac{\partial u_{i}}{\partial x_{i}} & =\frac{1}{z_{i}} f^{\prime}\left(\frac{x_{i}}{z_{i}}-1\right)-\frac{d_{i}}{\alpha_{i} x_{i}^{2}} g^{\prime}\left(\frac{d_{i}}{\alpha_{i} x_{i}}\right), \frac{\partial u_{i}}{\partial z_{i}}=-\frac{x_{i}}{z_{i}^{2}} f^{\prime}\left(\frac{x_{i}}{z_{i}}-1\right), \\
\frac{\partial w_{i}}{\partial x_{i}} & =\lambda w_{i} \frac{\partial u_{i}}{\partial x_{i}}, \quad \frac{\partial w_{i}}{\partial z_{i}}=\lambda w_{i} \frac{\partial u_{i}}{\partial z_{i}}
\end{aligned}
$$

Define $\beta:=f^{\prime}(0), \gamma:=g^{\prime}(r)$, and denote the FSS by $\mathbf{s}^{*}:=\left(\bar{x}_{1}, \bar{x}_{2}, \bar{x}_{1}, \bar{x}_{2}\right)=\left(\frac{d_{1}}{r \alpha_{1}}, \frac{d_{2}}{r \alpha_{2}}, \frac{d_{1}}{r \alpha_{1}}, \frac{d_{2}}{r \alpha_{2}}\right)$. At the FSS, the above expressions reduce to:

$$
\begin{aligned}
& \left.\frac{\partial u_{i}}{\partial x_{i}}\right|_{\mathbf{s}^{*}}=\frac{\beta-r \gamma}{\bar{x}_{i}},\left.\quad \frac{\partial u_{i}}{\partial z_{i}}\right|_{\mathbf{s}^{*}}=-\frac{\beta}{\bar{x}_{i}} \\
& \left.\frac{\partial w_{i}}{\partial x_{i}}\right|_{\mathbf{s}^{*}}=\lambda \bar{w} \frac{\beta-r \gamma}{\bar{x}_{i}},\left.\quad \frac{\partial w_{i}}{\partial z_{i}}\right|_{\mathbf{s}^{*}}=-\lambda \bar{w} \frac{\beta}{\bar{x}_{i}} .
\end{aligned}
$$

As a consequence:

$$
\begin{aligned}
& \left.\frac{\partial \tilde{F}_{1}}{\partial x_{1}}\right|_{\mathbf{s}^{*}}=1,\left.\quad \frac{\partial \tilde{F}_{1}}{\partial w_{1}}\right|_{\mathbf{s}^{*}}=\frac{\bar{x}_{1}\left(1-\bar{x}_{1}\right)}{\bar{w}}, \\
& \left.\frac{\partial \tilde{F}_{1}}{\partial x_{2}}\right|_{\mathbf{s}^{*}}=0,\left.\quad \frac{\partial \tilde{F}_{1}}{\partial w_{2}}\right|_{\mathbf{s}^{*}}=-\frac{\bar{x}_{1} \bar{x}_{2}}{\bar{w}}
\end{aligned}
$$

and

$$
\begin{aligned}
& \left.\frac{\partial \tilde{F}_{2}}{\partial x_{1}}\right|_{\mathbf{s}^{*}}=0,\left.\quad \frac{\partial \tilde{F}_{2}}{\partial w_{1}}\right|_{\mathbf{s}^{*}}=-\frac{\bar{x}_{1} \bar{x}_{2}}{\bar{w}}, \\
& \left.\frac{\partial \tilde{F}_{2}}{\partial x_{2}}\right|_{\mathbf{s}^{*}}=1,\left.\quad \frac{\partial \tilde{F}_{2}}{\partial w_{2}}\right|_{\mathbf{s}^{*}}=\frac{\bar{x}_{2}\left(1-\bar{x}_{2}\right)}{\bar{w}} .
\end{aligned}
$$

Finally, the (total) derivatives (31) and (32) are as follows:

$$
\begin{aligned}
& \left.\frac{\partial F_{1}}{\partial x_{1}}\right|_{\mathbf{s}^{*}}=1+\lambda\left(1-\bar{x}_{1}\right)(\beta-r \gamma),\left.\quad \frac{\partial F_{1}}{\partial x_{2}}\right|_{\mathbf{s}^{*}}=-\lambda \bar{x}_{1}(\beta-r \gamma), \\
& \left.\frac{\partial F_{1}}{\partial z_{1}}\right|_{\mathbf{s}^{*}}=-\lambda \beta\left(1-\bar{x}_{1}\right),\left.\quad \frac{\partial F_{1}}{\partial z_{2}}\right|_{\mathbf{s}^{*}}=\lambda \beta \bar{x}_{1},
\end{aligned}
$$


and, symmetrically:

$$
\begin{aligned}
& \left.\frac{\partial F_{2}}{\partial x_{1}}\right|_{\mathbf{s}^{*}}=-\lambda \bar{x}_{2}(\beta-r \gamma),\left.\quad \frac{\partial F_{2}}{\partial x_{2}}\right|_{\mathbf{s}^{*}}=1+\lambda\left(1-\bar{x}_{2}\right)(\beta-r \gamma), \\
& \left.\frac{\partial F_{2}}{\partial z_{1}}\right|_{\mathbf{s}^{*}}=\lambda \beta \bar{x}_{2},\left.\quad \frac{\partial F_{2}}{\partial z_{2}}\right|_{\mathbf{s}^{*}}=-\lambda \beta\left(1-\bar{x}_{2}\right),
\end{aligned}
$$

It is possible to reduce the number of parameters by setting $\tilde{\beta}:=\lambda \beta, \tilde{\gamma}:=\lambda \gamma$. By further introducing the simplified notation $\tilde{\sigma}:=\tilde{\beta}-r \tilde{\gamma}:=\lambda(\beta-r \gamma)$, the Jacobian matrix at the FSS can be rewritten as:

$$
\mathbf{J}\left(\mathbf{s}^{*}\right)=\left(\begin{array}{cccc}
1+\tilde{\sigma}\left(1-\bar{x}_{1}\right) & -\tilde{\sigma} \bar{x}_{1} & -\tilde{\beta}\left(1-\bar{x}_{1}\right) & \tilde{\beta} \bar{x}_{1} \\
-\tilde{\sigma} \bar{x}_{2} & 1+\tilde{\sigma}\left(1-\bar{x}_{2}\right) & \tilde{\beta} \bar{x}_{2} & -\tilde{\beta}\left(1-\bar{x}_{2}\right) \\
1 & 0 & 0 & 0 \\
0 & 1 & 0 & 0
\end{array}\right) .
$$

One can show that the characteristic polynomial of $\mathbf{J}\left(\mathbf{s}^{*}\right)$ can be expressed as follows: ${ }^{17}$

$$
\begin{aligned}
\mathcal{P}(v) & :=\operatorname{Det}\left(\mathbf{J}\left(\mathbf{s}^{*}\right)-v \mathbf{I}\right)= \\
& v^{4}-[(1+\tilde{\sigma})+(1+\tilde{\sigma} \bar{x})] v^{3} \\
& +[\tilde{\beta}(1+\bar{x})+(1+\tilde{\sigma})(1+\tilde{\sigma} \bar{x})] v^{2}-[\tilde{\beta} \bar{x}(1+\tilde{\sigma})+\tilde{\beta}(1+\tilde{\sigma} \bar{x})] v+\tilde{\beta}^{2} \bar{x}
\end{aligned}
$$

where $\bar{x}:=1-\bar{x}_{1}-\bar{x}_{2}$ represents the residual proportion of investors in the bond market at the steady state. Finally, one can check that $\mathcal{P}(v)$ can be factorized as the product of two second-degree polynomials, namely:

$$
\mathcal{P}(v)=\mathcal{P}_{1}(v) \mathcal{P}_{2}(v)=\left[v^{2}-(1+\tilde{\sigma}) v+\tilde{\beta}\right]\left[v^{2}-(1+\tilde{\sigma} \bar{x}) v+\tilde{\beta} \bar{x}\right] .
$$

The four characteristic roots of $\mathcal{P}(v)$ correspond to the two characteristic roots of $\mathcal{P}_{1}(v)$ - say $v_{1}$ and $v_{2}$ - and to the two roots of $\mathcal{P}_{2}(v)$ - say $v_{3}$ and $v_{4}$. This simplified structure of the linearized model at the FSS allows us to investigate the stability and possible bifurcations of the FSS in two steps, by relying on standard techniques for two-dimensional systems. In particular (see, e.g. Medio and Lines 2001), roots $v_{1}$ and $v_{2}$ are jointly less than one in modulus if and only if:

$$
\mathcal{P}_{1}(1)>0, \quad \mathcal{P}_{1}(-1)>0, \quad 1-\mathcal{P}_{1}(0)>0 .
$$

Likewise, a necessary and sufficient condition for $v_{3}$ and $v_{4}$ to be smaller than one in modulus is:

$$
\mathcal{P}_{2}(1)>0, \quad \mathcal{P}_{2}(-1)>0, \quad 1-\mathcal{P}_{2}(0)>0 .
$$

Conditions (33) and (34) jointly result in:

$$
\left\{\begin{array} { c } 
{ \tilde { \beta } - \tilde { \sigma } > 0 } \\
{ 2 + \tilde { \beta } + \tilde { \sigma } > 0 } \\
{ \tilde { \beta } < 1 }
\end{array} \quad \text { and } \quad \left\{\begin{array}{c}
(\tilde{\beta}-\tilde{\sigma}) \bar{x}>0 \\
2+(\tilde{\beta}+\tilde{\sigma}) \bar{x}>0 \\
\tilde{\beta} \bar{x}<1
\end{array},\right.\right.
$$

\footnotetext{
${ }^{17}$ This can be checked, e.g. by co-factor expansion of the fourth row of $\operatorname{Det}\left(\mathbf{J}\left(\mathbf{s}^{*}\right)-v \mathbf{I}\right)$.
} 
where the set of conditions on the left (right) correspond to polynomial $\mathcal{P}_{1}\left(\mathcal{P}_{2}\right)$, and where $0<\bar{x}<1$. Since $\tilde{\beta}-\tilde{\sigma}=\lambda r \gamma>0$, the first condition is always true for both sets. Let us now turn to the second condition. If $\tilde{\beta}+\tilde{\sigma}=\lambda(2 \beta-r \gamma) \geq 0$, the second condition is satisfied for both sets. Therefore, $\tilde{\beta}+\tilde{\sigma}<0$, i.e. $\beta<r \gamma / 2$, represents a necessary condition for the second condition to be violated. If this is the case, the second condition on the left turns out to be more restrictive than its counterpart on the right. Finally, the third condition on the left is more restrictive than the corresponding condition on the right. To summarize, the stability domain of the FSS is determined, in terms of the original parameters, by the following double inequality:

$$
\frac{r}{2} \lambda \gamma-1<\lambda \beta<1
$$

where violation of the inequality on the left leads to a period-doubling bifurcation (since it corresponds to $\mathcal{P}_{1}(-1) \leq 0$ ), whereas violation of the inequality on the right results in a Neimark-Sacker bifurcation (as it corresponds to $\mathcal{P}_{1}(0) \geq 1$ ).

A4. Model dynamics and steady state in the case $N^{B}=0$.

Investors' herding behavior can have a marked impact on the dynamics of our model. Note that the replicator equations (22) and (23) imply that if there are no investors in the bond market in period $t-1$, i.e. $N_{t-1}^{B}=0$ or, equivalently, $N_{t-1}^{H}=1-N_{t-1}^{S}$, there will also be no investors in the bond market in period $t$, i.e. $N_{t}^{B}=0$ or, equivalently, $N_{t}^{H}=1-N_{t}^{S}$. This means that the subset of the state space characterized by the condition of "no investors in the bond market' is trapping ${ }^{18}$ under the iteration of the evolutionary equations (22)-(23). There are two possibilities that can produce this outcome. First, the model parameters may be such that $r<r_{l}:=D / \alpha^{S}+R / \alpha^{H}$, i.e. $\bar{N}^{S}+\bar{N}^{H}>1$. Then $N_{t}^{B}$ is endogenously driven down to zero while the sum of $N_{t}^{S}$ and $N_{t}^{H}$ approaches one. An economic example of this could be low interest rates that render the bond market so unattractive that all investors move to stock and housing markets. Second, even if $\bar{N}^{S}+\bar{N}^{H}<1$, exogenous shocks may simply turn $N_{t}^{B}=0$ in some period $t$, automatically yielding $N_{t}^{S}+N_{t}^{H}=1$.

The model is then represented by a second-order nonlinear equation or, equivalently, by a two-dimensional nonlinear map, as follows:

$$
N_{t}^{S}=\frac{N_{t-1}^{S}}{N_{t-1}^{S}+\left(1-N_{t-1}^{S}\right) \exp \left[\lambda\left(A_{t-1}^{H}-A_{t-1}^{S}\right)\right]}
$$

where:

$$
A_{t-1}^{H}-A_{t-1}^{S}=f\left(\frac{N_{t-2}^{S}-N_{t-1}^{S}}{1-N_{t-2}^{S}}\right)-f\left(\frac{N_{t-1}^{S}-N_{t-2}^{S}}{N_{t-2}^{S}}\right)+g\left(\frac{R}{\alpha^{H}\left(1-N_{t-1}^{S}\right)}\right)-g\left(\frac{D}{\alpha^{S} N_{t-1}^{S}}\right) .
$$

\footnotetext{
${ }^{18}$ More formally, denote by $T$ the 4-dimensional map in variables $N^{S}, N^{H}, Z^{S}, Z^{H}$ (where $Z^{S}, Z^{H}$ represent the one-period lagged values of $N^{S}, N^{H}$ ) associated with our model, and denote by $\mathcal{S}$ the subset of the phase space corresponding to the case of no investors in the bond market, namely, $\mathcal{S}:=\left(N^{S}, 1-N^{S}, Z^{S}, 1-Z^{S}\right)$, $0<N^{S}<1,0<Z^{S}<1$. This subset is trapping in that, if $\mathbf{x} \in \mathcal{S}$, then $T(\mathbf{x}) \in \mathcal{S}$ or, put differently, the dynamics cannot escape $\mathcal{S}$. In fact, in order for the system to be trapped in subset $\mathcal{S}$, it is enough that it starts from a point in the broader set $\mathcal{S}^{\prime}$, where $\mathcal{S}^{\prime}=\left(N^{S}, 1-N^{S}, Z^{S}, Z^{H}\right), 0<N^{S}<1,0<Z^{S}<1,0<Z^{H}<1$, $Z^{S}+Z^{H} \leq 1$, as one can easily check.
} 
Therefore, the dynamics at the steady state need to satisfy (we now use the "^" accent to denote steady-state quantities):

$$
\hat{A}^{H}-\hat{A}^{S}=g\left(\frac{R}{\alpha^{H}\left(1-\hat{N}^{S}\right)}\right)-g\left(\frac{D}{\alpha^{S} \hat{N}^{S}}\right),
$$

and

$$
\hat{N}^{S}+\left(1-\hat{N}^{S}\right) \exp \left[\lambda\left(\hat{A}^{H}-\hat{A}^{S}\right)\right]=1 .
$$

The latter condition, along with the identity $\hat{N}^{S}+\left(1-\hat{N}^{S}\right)=1$, necessarily implies $\hat{A}^{H}=\hat{A}^{S}$. It follows that, in the case $N^{B}=0$, the dynamical system admits a non-fundamental steady state (NFSS), defined by the condition:

$$
\frac{R}{\alpha^{H}\left(1-\hat{N}^{S}\right)}=\frac{D}{\alpha^{S} \hat{N}^{S}},
$$

which results in the following equilibrium proportions:

$$
\hat{N}^{S}=\frac{\alpha^{H} D}{\alpha^{S} R+\alpha^{H} D}, \quad \hat{N}^{H}=\frac{\alpha^{S} R}{\alpha^{S} R+\alpha^{H} D},
$$

while the steady-state prices are defined as:

$$
\hat{P}^{S}=\frac{\alpha^{S} \alpha^{H} D}{\alpha^{S} R+\alpha^{H} D}, \quad \hat{P}^{H}=\frac{\alpha^{H} \alpha^{S} R}{\alpha^{S} R+\alpha^{H} D} .
$$

While the two markets still have identical 'dividend-price' ratios $D / \hat{P}^{S}$ and $R / \hat{P}^{H}$ at the NFSS, such quantities turn out to be different to the interest rate $r$, which plays no role in the development of the dynamical system anymore. When $\bar{N}^{S}+\bar{N}^{H}>1$, a comparison of $\bar{P}^{S}$ and $\bar{P}^{H}$ in (16) with $\hat{P}^{S}$ and $\hat{P}^{H}$ in (38) reveals that $\bar{P}^{S}>\hat{P}^{S}$ and $\bar{P}^{H}>\hat{P}^{H}$, i.e. the NFSS is always below the (unfeasible) FSS. The economic reason for this is that there are insufficient investors, or the investors have insufficient funds to push steady-state prices to their proper fundamental values. We have here another example where limits of arbitrage prevent the formation of efficient prices (Shleifer and Vishny 1997). However, when $\bar{N}^{S}+\bar{N}^{H}<1$, we obtain $\bar{P}^{S}<\hat{P}^{S}$ and $\bar{P}^{H}<\hat{P}^{H}$, i.e. if all investors enter stock and housing markets at some time $\left(N_{t}^{B}=0\right)$, we observe bubble equilibria. Now we face a situation in which too much wealth is put into stock and housing markets. Although our numerical experiments suggest that this is not a 'stable' situation for the deterministic model, we may argue that such bubble equilibria will last as long as investors do not want to exit the overvalued stock and housing markets (i.e. as long as there are no shocks in the opposite direction). Moreover, the dividend-price and rent-price ratios at the NFSS differ from the fundamental case in two more respects. First, they depend on behavioral parameters (individual demand) and price reaction. Second, the steady state in each market depends on the characteristics of both markets. Finally, note that the NFSS always exists. Therefore, as summarized in Appendix A1, if the complete model admits an inner FSS (which occurs for $r>r_{l}:=D / \alpha^{S}+R / \alpha^{H}$ ), the NFSS (37) represents a further steady state for the four-dimensional model, too.

For the dynamics restricted to the case $N^{B}=0$, the steady state may be stable or become unstable, either via a Neimark-Sacker bifurcation or a Flip bifurcation. Below we outline 
the derivation of the stability conditions for the NFSS (37), regarded as the steady state of the reduced model (36). With the simplified notation introduced in Appendix A3, the twodimensional map has now the following structure:

$$
x_{1}^{\prime}=F_{1}\left(x_{1}, z_{1}\right), \quad z_{1}^{\prime}=x_{1},
$$

where function $F_{1}$, representing the exponential replicator dynamics (36), can be rewritten as:

$$
F_{1}\left(x_{1}, z_{1}\right)=\frac{x_{1} w_{1}\left(x_{1}, z_{1}\right)}{x_{1}\left[w_{1}\left(x_{1}, z_{1}\right)-w_{2}\left(1-x_{1}, 1-z_{1}\right)\right]+w_{2}\left(1-x_{1}, 1-z_{1}\right)},
$$

with:

$$
\begin{aligned}
& w_{1}\left(x_{1}, z_{1}\right)=\exp \left[\lambda u_{1}\left(x_{1}, z_{1}\right)\right], \quad w_{2}\left(1-x_{1}, 1-z_{1}\right)=\exp \left[\lambda u_{2}\left(1-x_{1}, 1-z_{1}\right)\right] \\
& u_{1}\left(x_{1}, z_{1}\right)=f\left(\frac{x_{1}-z_{1}}{z_{1}}\right)+g\left(\frac{d_{1}}{\alpha_{1} x_{1}}\right), \quad u_{2}\left(1-x_{1}, 1-z_{1}\right)=f\left(\frac{z_{1}-x_{1}}{1-z_{1}}\right)+g\left(\frac{d_{2}}{\alpha_{2}\left(1-x_{1}\right)}\right) .
\end{aligned}
$$

Denote the NFSS (i.e. the fixed point of map (39)) by $\mathbf{c}^{*}:=\left(\hat{x}_{1}, \hat{x}_{1}\right)$, where:

$$
\hat{x}_{1}=\frac{\alpha_{2} d_{1}}{\alpha_{1} d_{2}+\alpha_{2} d_{1}} .
$$

Since $1-\hat{x}_{1}=\hat{x}_{2}=\alpha_{1} d_{2} /\left(\alpha_{1} d_{2}+\alpha_{2} d_{1}\right)$, the two markets have identical dividend-price ratios at the NFSS, namely:

$$
\hat{\zeta}_{1}:=\frac{d_{1}}{\alpha_{1} \hat{x}_{1}}=\frac{\alpha_{1} d_{2}+\alpha_{2} d_{1}}{\alpha_{1} \alpha_{2}}=\frac{d_{2}}{\alpha_{2}\left(1-\hat{x}_{1}\right)}:=\hat{\zeta}_{2},
$$

and the common dividend-price ratio $\hat{\zeta}_{1}=\hat{\zeta}_{2}$ is equal to the interest rate threshold $r_{l}$ defined by equation (17), as discussed in Section 3.1. As a consequence, at the NFSS, $u_{1}\left(\hat{x}_{1}, \hat{z}_{1}\right)=$ $u_{2}\left(1-\hat{x}_{1}, 1-\hat{z}_{1}\right)=f(0)+g\left(r_{l}\right):=\hat{u}$ and $w_{1}\left(\hat{x}_{1}, \hat{z}_{1}\right)=w_{2}\left(1-\hat{x}_{1}, 1-\hat{z}_{1}\right)=\exp (\lambda \hat{u}):=\hat{w}$. Finally, the denominator of (40) at the NFSS is also equal to the above-defined quantity $\hat{w}$.

Using the above results, and following similar steps as in Appedix A3, one obtains the following Jacobian matrix at the NFSS:

$$
\mathbf{J}\left(\mathbf{c}^{*}\right)=\left(\begin{array}{cc}
\frac{\partial F_{1}}{\partial x_{1}}\left(\mathbf{c}^{*}\right) & \frac{\partial F_{1}}{\partial z_{1}}\left(\mathbf{c}^{*}\right) \\
1 & 0
\end{array}\right)=\left(\begin{array}{cc}
1+\lambda\left(\beta-r_{l} \hat{\gamma}\right) & -\lambda \beta \\
1 & 0
\end{array}\right)
$$

where $\beta:=f^{\prime}(0), \hat{\gamma}:=g^{\prime}\left(r_{l}\right)$. The characteristic polynomial of $\mathbf{J}\left(\mathbf{c}^{*}\right)$, namely:

$$
\mathcal{Q}(v)=v^{2}-\left[1+\lambda\left(\beta-r_{l} \hat{\gamma}\right)\right] v+\lambda \beta,
$$

has roots smaller than one in modulus iff:

$$
\lambda r_{l} \hat{\gamma}>0, \quad 2(1+\lambda \beta)-\lambda r_{l} \hat{\gamma}>0, \quad \lambda \beta<1
$$

where, similarly to the general case, violation of the second (third) condition implies a Flip bifurcation (a Neimark-Sacker bifurcation). The local stability conditions of the NFSS in the 
two-dimensional model are formally the same conditions as for the FSS in the four-dimensional model. In particular, the Neimark-Sacker bifurcation still occurs for $\beta>1 / \lambda$ whereas, in general, the Flip bifurcation thresholds for parameters $\hat{\gamma}:=g^{\prime}\left(r_{l}\right)$ and $\gamma:=g^{\prime}(r)$ may be different.

\section{A5. An extended model with endogenous housing supply and rent}

We consider the basic setup described in Section 2.2 but assume that the housing stock, $X^{H}$, evolves over time due to depreciation (at rate $\delta$ per period) and new constructions, where the latter depend positively on the price level in the previous period, namely:

$$
X_{t}^{H}=(1-\delta) X_{t-1}^{H}+h\left(P_{t-1}^{H}\right), \quad 0<\delta<1
$$

where $h^{\prime}>0$. Thus, market clearing in the housing market, $D_{t}^{H}=X_{t}^{H}$, yields

$$
P_{t}^{H}=\alpha_{t}^{H} N_{t}^{H}
$$

where $\alpha_{t}^{H}:=I^{H} / X_{t}^{H}>0$. Moreover, we assume that rent $R_{t}$ adjusts with respect to demand and supply in the rental market. By assuming that (i) the amount of housing available for rent is proportional to the stock of housing, (ii) demand for housing consumption is negatively related to the rent level, and (iii) the rental market clears in each period, one obtains (see, e.g. Wheaton 1999, Dieci and Westerhoff 2016):

$$
R_{t}=q\left(X_{t}^{H}\right), \quad q^{\prime}<0 .
$$

As a consequence, the attractiveness of the housing market in period $t$ is given by

$$
A_{t}^{H}=f\left(\rho_{t}^{H}\right)+\gamma \frac{R_{t}}{P_{t}^{H}}
$$

where:

$$
\rho_{t}^{H}:=\frac{P_{t}^{H}-P_{t-1}^{H}}{P_{t-1}^{H}}=\frac{\alpha_{t}^{H} N_{t}^{H}}{\alpha_{t-1}^{H} N_{t-1}^{H}}-1=\frac{X_{t-1}^{H}}{X_{t}^{H}} \frac{N_{t}^{H}}{N_{t-1}^{H}}-1
$$

is the relative price change in the housing market in period $t$. Note that $\rho_{t}^{H}$ is no longer equal to the relative change in the number of investors in the housing market, since the marginal price impact per investor, $\alpha^{H}$, is now time-varying due to the varying housing supply. The dynamical system governing the model with endogenous housing stock and rent is still based on the exponential replicator equations (5)-(7), where $X_{t}^{H}, P_{t}^{H}, R_{t}, A_{t}^{H}$ and $\rho_{t}^{H}$ respond to the modified definitions (41)-(45).

The fundamental steady state (FSS) of the model is again characterized by a perfect balance between the three markets in terms of their attractiveness. By denoting steady-state quantities with an overbar, the no-arbitrage condition:

$$
\bar{A}^{S}=\bar{A}^{H}=A^{B},
$$

yields, in particular, $\bar{\alpha}^{H}=I^{H} / \bar{X}^{H}$ and:

$$
\frac{D}{\alpha^{S} \bar{N}^{S}}=\frac{\bar{R}}{\bar{\alpha}^{H} \bar{N}^{H}}=r
$$


from which:

$$
\bar{N}^{S}=\frac{D}{\alpha^{S} r}, \quad \bar{P}^{S}=\frac{D}{r}, \quad \bar{N}^{H}=\frac{\bar{R}}{\bar{\alpha}^{H} r}, \quad \bar{P}^{H}=\frac{\bar{R}}{r} .
$$

Finally, in equation (46), steady-state housing stock $\bar{X}^{H}$ (which determines the 'impact' coefficient $\bar{\alpha}^{H}=I^{H} / \bar{X}^{H}$ ) and steady-state rent $\bar{R}$ need to satisfy the following conditions (from (41) and (43)):

$$
h\left(\bar{P}^{H}\right)=\delta \bar{X}^{H}, \quad r \bar{P}^{H}=q\left(\bar{X}^{H}\right) .
$$

Under standard isoelastic specifications of functions $h$ and $q$, namely:

$$
\begin{aligned}
h\left(P_{t-1}^{H}\right) & =\eta_{0}\left(P_{t-1}^{H}\right)^{\eta}, \quad \eta_{0} \geq 0, \quad \eta>0, \\
q\left(X_{t}^{H}\right) & =\xi_{0}\left(X_{t}^{H}\right)^{-\xi}, \quad \xi_{0} \geq 0, \quad \xi>0,
\end{aligned}
$$

conditions (47), rewritten in terms of steady-state rent $\bar{R}=r \bar{P}^{H}$, are given as follows:

$$
\eta_{0}\left(\frac{\bar{R}}{r}\right)^{\eta}=\delta \bar{X}^{H}, \quad \bar{R}=\xi_{0}\left(\bar{X}^{H}\right)^{-\xi} .
$$

We select a parametrization that allows us to compare the results to our baseline case, where rent $R$ and market impact $\alpha^{H}$ are fixed parameters. By making steady-state rent $\bar{R}$ equal to parameter $R$ of the baseline case, from condition (48) one obtains steady-state stock $\bar{X}^{H}$ as:

$$
\bar{X}^{H}=\frac{\eta_{0}}{\delta}\left(\frac{\bar{R}}{r}\right)^{\eta} .
$$

Next, since parameter $I^{H}$ can be selected arbitrarily in our model, we choose $I^{H}=\alpha^{H} \bar{X}^{H}$, where $\alpha^{H}$ is the desired level of parameter $\alpha^{H}$ (in particular, $\alpha^{H}=1$ in our baseline parameter setting). Finally, from the steady-state condition $\bar{R}=\xi_{0}\left(\bar{X}^{H}\right)^{-\xi}$, one obtains $\xi_{0}=\bar{R}\left(\bar{X}^{H}\right)^{\xi}$, so that function $q\left(X_{t}^{H}\right)$ is conveniently rescaled as follows:

$$
R_{t}=q\left(X_{t}^{H}\right)=R\left(\frac{X_{t}^{H}}{\bar{X}^{H}}\right)^{-\xi},
$$

which has the value $\bar{R}=R$ at the steady state.

One can check that the resulting dynamic model is six-dimensional. Numerical investigation of the model under different parameter constellations shows that the key qualitative insights drawn from the baseline case are robust to more general and flexible assumptions about the functioning of the housing market. In particular, Figure 7 represents the dynamic behavior of the extended model in the Neimark-Sacker scenario, under the same parameter setting used in Section 3.2 and assuming, additionally, $\delta=0.005, \eta_{0}=1, \eta=2$ and $\xi=2$. Comparing the bifurcations diagrams in the top panels with those in the top panels of Figure 2 reveals that the extrapolation parameter $\beta$ can again destabilize the steady state and produce quasiperiodic motion with price fluctuations of increasing amplitude (although the fluctuations in the housing market appear to be damped due to a sufficiently flexible housing supply). Also, the NeimarkSacker bifurcation value for parameter $\beta$ seems to be the same as in the baseline case. The price time paths and phase plots in the bottom panels can be compared with those in the top panels of Figure 3. As can be seen, the two markets still evolve countercyclically to each other. Such qualitative features depend largely on investors' modes of behavior and switching across markets and seem to be sufficiently robust to possible extensions of our baseline setup towards a more realistic description of the characteristic features of stock and housing markets. 

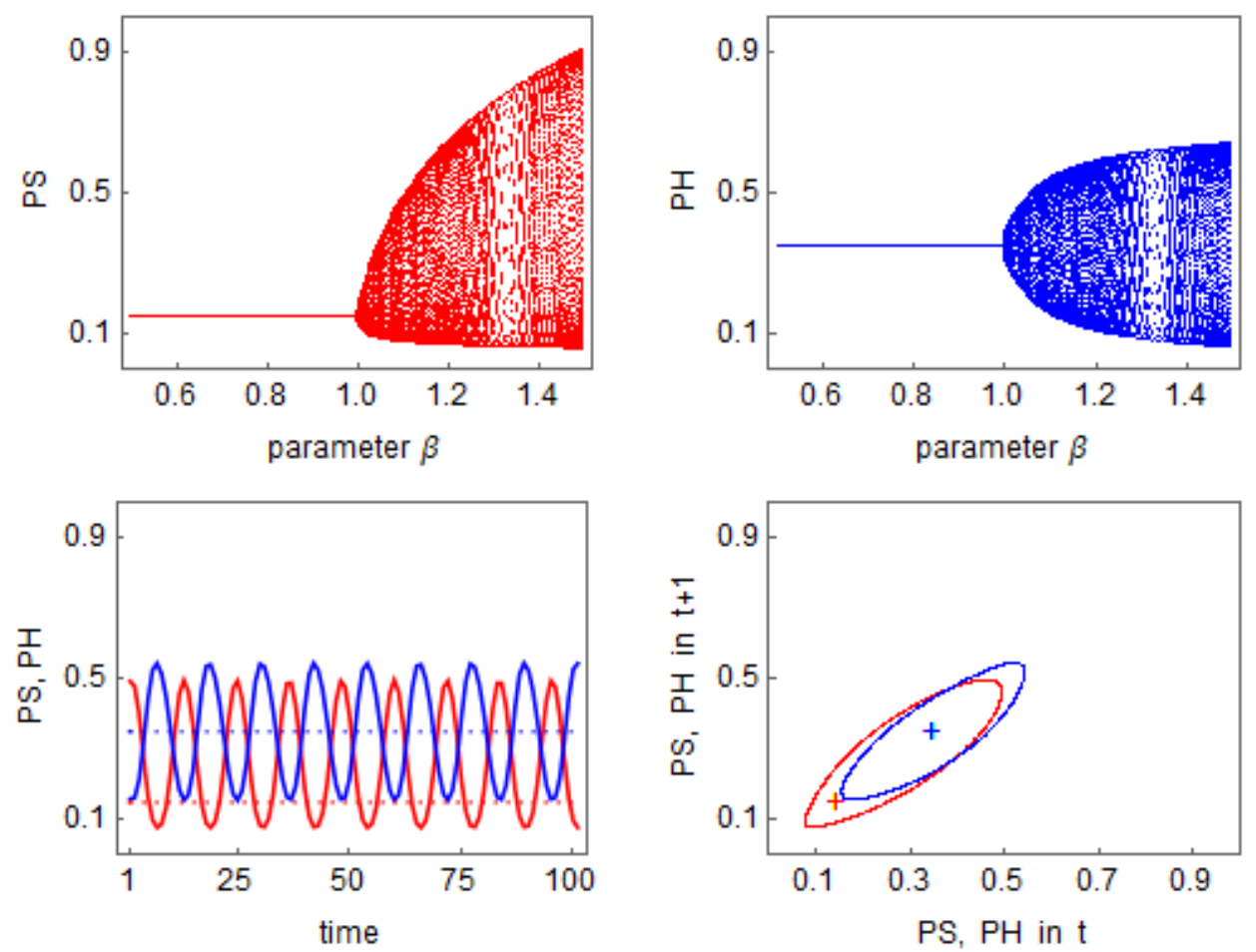

Figure 7: The dynamics of the model with time varying rents and housing supply in the 'Neimark-Sacker scenario'. The bifurcation plots in the top left (top right) panel depict the asymptotic behavior of $P^{S}\left(P^{H}\right)$ for $\beta$ ranging from 0.5 to 1.5. New parameters specific to the extended models are: $\delta=0.005, \eta=2, \eta_{0}=1, \xi=2$, while other parameters are as in our base selection specified at the beginning of Section 3.2. The bottom left (bottom right) panel shows the dynamics in the time domain (in the phase space) for prices in the stock market (red) and housing market (blue). The dotted lines and crosses indicate the markets' steady-state values. Parameter as in top panels with $\beta=1.1$. 

101 Mafaïzath A. Fatoke-Dato, Impact of an educational demand-and-supply policy on girls' education in West Africa: Heterogeneity in income, school environment and ethnicity, June 2015

102 Mafaïzath A. Fatoke-Dato, Impact of income shock on children's schooling and labor in a West African country, June 2015

103 Noemi Schmitt, Jan Tuinstra and Frank Westerhoff, Side effects of nonlinear profit taxes in an evolutionary market entry model: abrupt changes, coexisting attractors and hysteresis problems, August 2015.

104 Noemi Schmitt and Frank Westerhoff, Evolutionary competition and profit taxes: market stability versus tax burden, August 2015.

105 Lena Dräger and Christian R. Proaño, Cross-Border Banking and Business Cycles in Asymmetric Currency Unions, November 2015.

106 Christian R. Proaño and Benjamin Lojak, Debt Stabilization and Macroeconomic Volatility in Monetary Unions under Heterogeneous Sovereign Risk Perceptions, November 2015.

107 Noemi Schmitt and Frank Westerhoff, Herding behavior and volatility clustering in financial markets, February 2016

108 Jutta Viinikainen, Guido Heineck, Petri Böckerman, Mirka Hintsanen, Olli Raitakari and Jaakko Pehkonen, Born Entrepreneur? Adolescents’ Personality Characteristics and Self-Employment in Adulthood, March 2016

109 Stefanie P. Herber and Michael Kalinowski, Non-take-up of Student Financial Aid: A Microsimulation for Germany, April 2016

110 Silke Anger and Daniel D. Schnitzlein, Cognitive Skills, Non-Cognitive Skills, and Family Background: Evidence from Sibling Correlations, April 2016

111 Noemi Schmitt and Frank Westerhoff, Heterogeneity, spontaneous coordination and extreme events within large-scale and small-scale agent-based financial market models, June 2016

112 Benjamin Lojak, Sentiment-Driven Investment, Non-Linear Corporate Debt Dynamics and Co-Existing Business Cycle Regimes, July 2016

113 Julio González-Díaz, Florian Herold and Diego Domínguez, Strategic Sequential Voting, July 2016

114 Stefanie Yvonne Schmitt, Rational Allocation of Attention in Decision-Making, July 2016

115 Florian Herold and Christoph Kuzmics, The evolution of taking roles, September 2016. 
116 Lisa Planer-Friedrich and Marco Sahm, Why Firms Should Care for All Consumers, September 2016.

117 Christoph March and Marco Sahm, Asymmetric Discouragement in Asymmetric Contests, September 2016.

118 Marco Sahm, Advance-Purchase Financing of Projects with Few Buyers, October 2016.

119 Noemi Schmitt and Frank Westerhoff, On the bimodality of the distribution of the S\&P 500’s distortion: empirical evidence and theoretical explanations, January 2017

120 Marco Sahm, Risk Aversion and Prudence in Contests, March 2017

121 Marco Sahm, Are Sequential Round-Robin Tournaments Discriminatory?, March 2017

122 Noemi Schmitt, Jan Tuinstra and Frank Westerhoff, Stability and welfare effects of profit taxes within an evolutionary market interaction model, May 2017

123 Johanna Sophie Quis and Simon Reif, Health Effects of Instruction Intensity - Evidence from a Natural Experiment in German High-Schools, May 2017

124 Lisa Planer-Friedrich and Marco Sahm, Strategic Corporate Social Responsibility, May 2017

125 Peter Flaschel, Matthieu Charpe, Giorgos Galanis, Christian R. Proaño and Roberto Veneziani, Macroeconomic and Stock Market Interactions with Endogenous Aggregate Sentiment Dynamics, May 2017

126 Christian Menden and Christian R. Proaño, Dissecting the Financial Cycle with Dynamic Factor Models, May 2017

127 Christoph March and Marco Sahm, Contests as Selection Mechanisms: The Impact of Risk Aversion, July 2017

128 Ivonne Blaurock, Noemi Schmitt and Frank Westerhoff, Market entry waves and volatility outbursts in stock markets, August 2017

129 Christoph Laica, Arne Lauber and Marco Sahm, Sequential Round-Robin Tournaments with Multiple Prizes, September 2017

130 Joep Lustenhouwer and Kostas Mavromatis, Fiscal Consolidations and Finite Planning Horizons, December 2017

131 Cars Hommes and Joep Lustenhouwer, Managing Unanchored, Heterogeneous Expectations and Liquidity Traps, December 2017

132 Cars Hommes, Joep Lustenhouwer and Kostas Mavromatis, Fiscal Consolidations and Heterogeneous Expectations, December 2017

133 Roberto Dieci, Noemi Schmitt and Frank Westerhoff, Interactions between stock, bond and housing markets, January 2018 